\title{
REALIZATION OF AUTOREGRESSIVE EQUATIONS IN PENCIL AND DESCRIPTOR FORM*
}

\author{
M. KUIJPER $†$ AND J. M. SCHUMACHER $\ddagger$
}

\begin{abstract}
A linear system described by autoregressive equations with a given input/output structure cannot be transformed to standard state-space form if the implied input/output relation is nonproper. Instead, a realization in descriptor form must be used. In this paper, it is shown how to obtain minimal descriptor realizations from autoregressive equations without separating finite and infinite frequencies, and without going through a reduction process. External equivalence is used, so that even situations in which there is no transfer matrix can be considered. The approach is based on the so-called pencil representation of linear systems, and it is shown that there is a natural realization of autoregressive equations in pencil form. In this way, the link between the realization theories of Willems and Fuhrmann can also be clarified.
\end{abstract}

Key words. linear systems, autoregressive equations, descriptor form, pencil representation, realization, external equivalence

AMS(MOS) subject classifications. 93B15, 93B20

1. Introduction and preliminaries. In this paper, we study methods for obtaining state representations for linear systems given by higher-order equations in external variables, with special attention to the so-called "nonproper" situation. Suppose that relations between input variables $u$ and output variables $y$ are specified by equations of the form

$$
R_{1}(\sigma) y+R_{2}(\sigma) u=0
$$

where $R_{1}(\sigma)$ and $R_{2}(\sigma)$ are polynomial matrices, $\sigma$ denotes differentiation or shift (depending on whether we work in continuous time or in discrete time), and $y$ and $u$ are functions of time. Here, as well as below, the time argument is suppressed to alleviate the notation. The argument $\sigma$ will sometimes be replaced by $\lambda$ or $s ; \lambda$ denotes a formal parameter, whereas $s$ is used as a complex parameter and serves as default. Following the terminology of Willems [19], we will refer to (1.1) as a set of autoregressive equations. Inputs and outputs are jointly referred to as external variables, and (1.1) may be rewritten as

$$
R(\sigma) w=0
$$

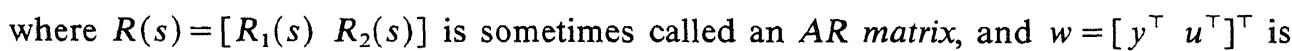
the vector of external variables. Of course, it is also possible to take (1.2) as a starting point, without distinction between "inputs" and "outputs" in the external variables. The behavior defined by (1.2) is the set of all time functions $w$ that satisfy (1.2). A behavior may also be specified by other means, for instance, by representations that involve auxiliary (internal) variables, such as the state representations to be defined below. Two representations will be said to be externally equivalent $[18]$ if their induced behaviors are the same. In this paper, we will be looking for minimal representations under external equivalence. In comparison with the notion of transfer equivalence, which has been used more commonly in realization theory, external equivalence is both stronger and more general-more general, because transfer equivalence can be

* Received by the editors January 16,1989 ; accepted for publication (in revised form) October 30, 1989.

$\uparrow$ Centre for Mathematics and Computer Science, P.O. Box 4079, 1009 AB Amsterdam, the Netherlands.

¥ Centre for Mathematics and Computer Science, P.O. Box 4079, 1009 AB Amsterdam, the Netherlands and Department of Economics, Tilburg University, P.O. Box 90153, 5000 LE Tilburg, the Netherlands. 
defined only for systems with a given input/output structure that is such that a transfer matrix exists, and stronger, because when both notions are applicable, external equivalence implies transfer equivalence but not the other way around. To avoid confusion, let us note that the notion of "external equivalence" as understood in [2] is different from the notion used here; for example, the systems $\dot{y}=\dot{u}$ and $y=u$ are equivalent in the sense of [2] but not in the sense of this paper.

The standard realization theory presupposes that the matrix $R_{1}(s)$ is square and nonsingular, and that $R_{1}^{-1}(s) R_{2}(s)$ is proper rational. Under these assumptions, it is well known that an equivalent representation can be found in the usual state-space form

$$
\sigma x=A x+B u, \quad y=C x+D u .
$$

A powerful and elegant method to obtain such a state-space realization was devised by Fuhrmann [5] who stated his result under transfer equivalence, and a similar procedure under external equivalence was given by Willems [19]. However, the standard assumptions mentioned above are not always satisfied. Examples of situations in which this occurs can be found, for instance, in circuit models [13], econometric models [11], and system inversion [7]. An often used modification of (1.3), that enables us to also cover these so-called nonproper situations, is the descriptor form [10]

$$
\sigma E x=A x+B u, \quad y=C x+D u
$$

where the matrix $E$ is not necessarily invertible. Algorithms to go from (1.1) to (1.4), which follow the line of [5], have been presented in [22] and [4]. Both papers work under transfer equivalence and so there is still the assumption that the matrix $R_{1}(s)$ is invertible. The realization procedure is then based on a decomposition of the transfer matrix $R_{1}^{-1}(s) R_{2}(s)$ into a strictly proper and a polynomial part. For the strictly proper part, a representation in standard state-space form is obtained by the usual means, and the polynomial part is realized in special descriptor form by using a modification of Fuhrmann's procedure; finally, the two realizations are put together again to create a representation in descriptor form.

One of the important uses of realization theory is the translation of properties of and statements about linear systems from polynomial terms to state-space terms and vice versa, as is extensively shown in [6]. The realization procedure for nonproper systems by cutting and pasting, as just described, is somewhat indirect, and is therefore less suitable for such translation purposes. In this paper, we will show how to obtain a realization in descriptor form without separation of finite and infinite frequencies. The realization will be obtained under external equivalence, and will be minimal in the appropriate sense. As an application, we will establish the relationships between basic indices associated with the representation (1.1) and with the representation (1.4). The realization procedure will be motivated along the lines of [19], and our discussion will also clarify the relationship between the realization algorithm in [19] and the one in [5].

The development below will be based on what we call the pencil representation of a linear system. This is a representation of the form

$$
\sigma G z=F z, \quad w=H z
$$

where $w$ is a vector of external variables containing both inputs and outputs, and $\sigma$ again denotes either differentiation or shift. A similar representation has been used before in [1], and pencil techniques in general are popular tools in numerical system theory (see, for instance, [16]). It may also be noted that the form (1.5) has been used for systems with partial differential equations in which control is exerted through the boundary conditions ("boundary control systems"; cf. [14]). 
Formally, a pencil representation is given by a six-tuple $(Z, X, W ; F, G, H)$ in which $W$ is the space of external variables, $Z$ is the space of internal variables, $X$ is the equation space, $F$ and $G$ are linear mappings from $Z$ to $X$, and $H$ is a linear mapping from $Z$ to $W$. We shall consider only pencil representations that are finitedimensional in the sense that both $\operatorname{dim} Z$ and $\operatorname{dim} X$ are finite. Also, $\operatorname{dim} W$ will always be finite. Two pencil representations $(Z, X, W ; F, G, H)$ and $(\tilde{Z}, \tilde{X}, W ; \tilde{F}, \tilde{G}$, $\tilde{H})$ will be called isomorphic if there exist isomorphisms $S: Z \rightarrow \tilde{Z}$ and $T: X \rightarrow \tilde{X}$ such that $\tilde{G}=T G S^{-1}, \tilde{F}=T F S^{-1}$, and $\tilde{H}=H S^{-1}$. The behavior given by a pencil representation is the set of all $w$ for which there exists a $z$ such that (1.5) holds. (One has to select suitable function classes here; this will be discussed later.) A pencil representation is said to be minimal (under external equivalence) if both $\operatorname{dim} Z$ and $\operatorname{dim} X$ are minimal in the class of equivalent representations. Let us quickly review what can be inferred about minimality of pencil representations from the existing literature.

Proposition 1.1. A pencil representation $(Z, X, W ; F, G, H)$ is minimal under external equivalence if and only if the following conditions hold:

(i) $G$ is surjective;

(ii) $\left[G^{\top} H^{\top}\right]^{\top}$ is injective;

(iii) the matrix $\left[s G^{\top}-F^{\top} H^{\top}\right]^{\top}$ has full column rank for all $s \in \mathbb{C}$.

Moreover, a minimal representation is unique up to isomorphism.

Proof. If $G$ is not surjective in a representation of the form (1.5), then "Step One" of the realization algorithm in [15] may be used to find an equivalent representation with a smaller equation space $X$. So in every minimal representation the mapping $G$ must be surjective. By a suitable choice of bases in $X$ and $Z$, a matrix representation of $G$ may then be given as $G=\left[\begin{array}{ll}I & 0\end{array}\right]$; with respect to these bases, write $F=\left[\begin{array}{ll}A B & B\end{array}\right]$, and $H=\left[\begin{array}{ll}C^{\prime} & D^{\prime}\end{array}\right]$. Writing $z$ correspondingly as a vector with components $\xi$ and $\eta$, the representation (1.5) takes the form

$$
\sigma \xi=A \xi+B \eta \quad w=C^{\prime} \xi+D^{\prime} \eta .
$$

The variable $\eta$ is known as the "driving variable" ([19]). It is known ([18, Thm. 4.5], $[19, \S 5],\left[15\right.$, Cor. 4.2]) that such a system is minimal if and only if $V^{*}\left(A, B, C^{\prime}\right.$, $\left.D^{\prime}\right)=\{0\}$ and $D^{\prime}$ is injective. The condition on $V^{*}$ and the injectivity of $D^{\prime}$ together imply that the associated system pencil

$$
\left(\begin{array}{cc}
s I-A & B \\
C^{\prime} & D^{\prime}
\end{array}\right)
$$

has full column rank for all $s$ (see $\left[8\right.$, p. 544]), so that (iii) holds. Because $D^{\prime}$ is injective, the matrix

$$
\left(\begin{array}{cc}
I & 0 \\
C^{\prime} & D^{\prime}
\end{array}\right)
$$

is injective, also; this implies (ii). Conversely, if the conditions (i)-(iii) hold, then it follows from (ii) and (iii) that the system pencil has full column rank for all $s$, so that $V^{*}$ in the equivalent state space form must be zero. The injectivity of $D^{\prime}$ in the equivalent state space form is immediate from (ii), by reversing the argument used above.

Now consider two minimal representations $(Z, X, W ; F, G, H)$ and $(\tilde{Z}, \tilde{X}, W$; $\tilde{F}, \tilde{G}, \tilde{H})$ of the same system. As above, both representations can be rewritten in driving-variable form; the resulting state-space representations will be denoted by $(A$, $\left.B, C^{\prime}, D^{\prime}\right)$ and $\left(\tilde{A}, \tilde{B}, \tilde{C}^{\prime}, \tilde{D}^{\prime}\right)$, respectively. Because these are minimal representations of the same behavior, it follows from Theorem 7.1 in [18] that there exist invertible 
mappings $Q$ and $R$ and a mapping $F$ such that $\tilde{A}=Q(A+B F) Q^{-1}, \tilde{B}=Q B R, \tilde{C}^{\prime}=$ $\left(C^{\prime}+D^{\prime} F\right) Q^{-1}$ and $\tilde{D}^{\prime}=D R$. So we can write the following equations:

$$
\begin{gathered}
{\left[\begin{array}{cc}
I & 0
\end{array}\right]=Q\left[\begin{array}{ll}
I & 0
\end{array}\right]\left(\begin{array}{cc}
Q^{-1} & 0 \\
F Q^{-1} & R
\end{array}\right)} \\
{\left[\begin{array}{cc}
\tilde{A} & \tilde{B}
\end{array}\right]=Q\left[\begin{array}{ll}
A & B
\end{array}\right]\left(\begin{array}{cc}
Q^{-1} & 0 \\
F Q^{-1} & R
\end{array}\right)} \\
{\left[\begin{array}{ll}
\tilde{C}^{\prime} & \tilde{D^{\prime}}
\end{array}\right]=\left[\begin{array}{ll}
C^{\prime} & D^{\prime}
\end{array}\right]\left(\begin{array}{cc}
Q^{-1} & 0 \\
F Q^{-1} & R
\end{array}\right) .}
\end{gathered}
$$

This shows that the two given representations are isomorphic.

Remark 1.2. It is not hard to see that if (i) of the above proposition holds and the matrix $\left[s G^{\top}-F^{\top} H^{\top}\right]^{\top}$ has full column rank (as a rational matrix), then condition (ii) holds if and only if $\left[s G^{\top}-F^{\top} H^{\top}\right]^{\top}$ has no zeros at infinity. So, items (ii) and

(iii) of the proposition may be replaced by the following two conditions:

(ii)' the matrix $\left[s G^{\top}-F^{\top} H^{\top}\right]^{\top}$ has full column rank;

(iii) $)^{\prime}$ the matrix $\left[s G^{\top}-F^{\top} H^{\top}\right]^{\top}$ has no zeros in the extended complex plane.

2. Pencil representations from a given behavior: discrete time. In this section, we will discuss the pencil representation for systems that are given directly through their (discrete-time) behavior. Here our treatment is close to the development in [19]; however, we emphasize the pencil representation rather than the driving-variable representation, and we derive some results that do not depend on the assumption that the behavior is closed in the topology of pointwise convergence.

Following the definition in [19], a linear, time-invariant, discrete-time behavior is a shift-invariant subspace of the space $W^{\mathbb{Z}_{+}}$of all functions from $\mathbb{Z}_{+}$to a vector space $W \simeq \mathbb{R}^{q}$. The following mappings are defined on $W^{\mathbb{Z}}$ : the shift

$$
\sigma:\left(w_{0}, w_{1}, \cdots\right) \mapsto\left(w_{1}, w_{2}, \cdots\right),
$$

the forward shift

$$
\sigma^{*}:\left(w_{0}, w_{1}, \cdots\right) \mapsto\left(0, w_{0}, w_{1}, \cdots\right),
$$

and the evaluation mapping at time 0

$$
\chi:\left(w_{0}, w_{1}, \cdots\right) \mapsto w_{0} .
$$

Now, let $\mathscr{B}$ be a given behavior. Following [19], we introduce the subspaces

$$
\mathscr{B}^{0}=\left\{w \in \mathscr{B} \mid\left(\sigma^{*}\right)^{k} w \in \mathscr{B} \forall k \geqq 0\right\}
$$

and

$$
\mathscr{B}^{1}=\left\{w \in \mathscr{B}^{0} \mid \chi w=0\right\}
$$

of $\mathscr{B}$. Intuitively, $\mathscr{B}^{0}$ contains the trajectories that start from the zero state; so the quotient space $\mathscr{B} / \mathscr{B}^{0}$ should be (isomorphic to) the state space. The quotient space $\mathscr{B}^{0} / \mathscr{B}^{1}$ describes the freedom that arises at each point in time because of the freedom we have in choosing a value of the input variable (or rather, a value of the "driving variable"). So, $\mathscr{B}^{0} / \mathscr{B}^{1}$ is the candidate for the space of driving variables. The following facts are trivially verified:

$$
\begin{aligned}
& \sigma \mathscr{B}^{1} \subset \mathscr{B}^{0} \\
& \mathscr{B}^{1} \subset \operatorname{ker} \chi
\end{aligned}
$$


Because of (2.6), we can properly define a mapping $M_{1}: \mathscr{B} / \mathscr{B}^{1} \rightarrow \mathscr{B} / \mathscr{B}^{0}$ by

$$
M_{1}: w \bmod \mathscr{B}^{1} \mapsto \sigma w \bmod \mathscr{B}^{0} .
$$

Because of $(2.7)$, there is also a mapping $M_{2}: \mathscr{B} / \mathscr{B}^{1} \rightarrow W$ defined by

$$
M_{2}: w \bmod \mathscr{B}{ }^{1} \mapsto \chi w .
$$

Furthermore, we introduce the projection mapping $M_{0}: \mathscr{B} / \mathscr{B}^{1} \rightarrow \mathscr{B} / \mathscr{B}^{0}$, defined simply by

$$
M_{0}: w \bmod \mathscr{B}^{1} \mapsto w \bmod \mathscr{B}^{0} .
$$

If elements of $\mathscr{B} / \mathscr{B}^{1}$ are seen as "state + driving variable," then $M_{0}$ deletes the driving variable. The mappings $M_{0}, M_{1}$, and $M_{2}$ could also have been introduced by requiring that Fig. 1 below commutes, where $\pi^{0}$ denotes projection modulo $\mathscr{B}^{0}$ and $\pi^{1}$ projection modulo $\mathscr{B}^{1}$.

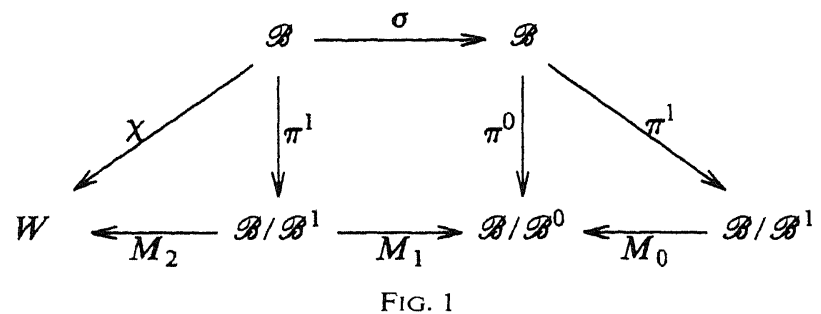

The discrete-time behavior described by a pencil representation such as (1.5) will be denoted by $\mathscr{B}_{\mathrm{p}}(Z, X, W ; F, G, H)$. More explicitly,

$$
\mathscr{B}_{\mathrm{p}}(Z, X, W ; F, G, H)=\left\{w: \mathbb{Z}_{+} \rightarrow W \mid \exists z: \mathbb{Z}_{+} \rightarrow \mathbb{Z} \text { s.t. } \sigma G z=F z \text { and } H z=w\right\} .
$$

We can now formulate the following proposition.

Proposition 2.1. For any linear, time-invariant, discrete-time behavior $\mathscr{B}$, we have

$$
\mathscr{B} \subset \mathscr{B}_{\mathrm{p}}\left(\mathscr{B} / \mathscr{B}^{1}, \mathscr{B} / \mathscr{B}^{0}, W ; M_{1}, M_{0}, M_{2}\right) \text {. }
$$

Proof. Take $w \in \mathscr{B}$. Define $z: \mathbb{Z}_{+} \rightarrow \mathscr{B} / \mathscr{B}^{1}$ by

$$
z_{k}=\pi^{1} \sigma^{k} w \text {. }
$$

From the definitions we easily verify that $\sigma M_{0} z=M_{1} z$ and that $M_{2} z=w$. This proves that $w \in \mathscr{B}_{\mathrm{p}}\left(\mathscr{B} / \mathscr{B}^{1}, \mathscr{B} / \mathscr{B}^{0}, W ; M_{1}, M_{0}, M_{2}\right)$.

The closure of a behavior $\mathscr{B}$ (in the topology of pointwise convergence) will be denoted by $\mathscr{B}^{\mathrm{cl}}$. A sequence $w$ belongs to $\mathscr{B}^{\mathrm{cl}}$ if and only if for every $k \geqq 0$ there exists a $\tilde{w} \in \mathscr{B}$ such that $w_{j}=\tilde{w}_{j}$ for all $0 \leqq j \leqq k$.

PROPOSITION 2.2. For any linear, time-invariant, discrete-time behavior $\mathscr{B}$, we have

$$
\mathscr{B}^{\mathrm{cl}} \supset \mathscr{B}_{\mathrm{p}}\left(\mathscr{B} / \mathscr{B}^{1}, \mathscr{B} / \mathscr{B}^{0}, W ; M_{1}, M_{0}, M_{2}\right) \text {. }
$$

Proof. Take $w \in \mathscr{B}_{\mathrm{p}}\left(\mathscr{B} / \mathscr{B}^{1}, \mathscr{B} / \mathscr{B}^{0}, W ; M_{1}, M_{0}, M_{2}\right)$, and let $z: \mathbb{Z}_{+} \rightarrow \mathscr{B} / \mathscr{B}^{1}$ be such that $\sigma M_{0} z=M_{1} z$ and $M_{2} z=w$. To show that $w \in \mathscr{B}^{\mathrm{cl}}$, we will prove by induction that for every $k$ there exists a $\tilde{w}^{k} \in \mathscr{B}$ such that $w_{i}=\tilde{w}_{i}^{k}$ for $0 \leqq i \leqq k$. First, let $\hat{w}^{k} \in \mathscr{B}$ be such that

Next, define $\tilde{w}^{k}$ by

For $0 \leqq i \leqq k$, we have

$$
z_{k}=\pi^{1} \hat{w}^{k}
$$

$$
\tilde{w}^{k}=\left(\hat{w}_{0}^{0}, \hat{w}_{0}^{1}, \cdots, \hat{w}_{0}^{k}, \hat{w}_{1}^{k}, \hat{w}_{2}^{k}, \cdots\right) .
$$

$$
w_{i}=M_{2} z_{i}=M_{2} \pi^{1} \hat{w}^{i}=\chi \hat{w}^{i}=\hat{w}_{0}^{i}=\tilde{w}_{i}^{k} .
$$


It remains to prove that $\tilde{w}^{k} \in \mathscr{B}$ for all $k$. For $k=0$, this is trivial since $\tilde{w}^{0}=\hat{w}^{0} \in \mathscr{B}$. Since

$$
\begin{aligned}
\tilde{w}^{k+1}-\tilde{w}^{k} & =\left(0,0, \cdots, 0, \hat{w}_{0}^{k+1}-\hat{w}_{1}^{k}, \hat{w}_{1}^{k+1}-\hat{w}_{2}^{k}, \cdots\right) \\
& =\left(\sigma^{*}\right)^{k}\left(\hat{w}^{k+1}-\sigma \hat{w}^{k}\right),
\end{aligned}
$$

the proof will follow by induction if we can show that $\hat{w}^{k+1}-\sigma \hat{w}^{k} \in \mathscr{B}^{0}$ for all $k$. But this follows from

$$
\pi^{0} \hat{w}^{k+1}=M_{0} \pi^{1} \hat{w}^{k+1}=M_{0} z_{k+1}=M_{1} z_{k}=M_{1} \pi^{1} \hat{w}^{k}=\pi^{0} \sigma \hat{w}^{k} .
$$

Corollary 2.3 [19]. If $\mathscr{B}=\mathscr{B}^{\mathrm{cl}}$, then $\mathscr{B}_{\mathrm{p}}\left(\mathscr{B} / \mathscr{B}^{1}, \mathscr{B} / \mathscr{B}^{0}, W ; M_{1}, M_{0}, M_{2}\right)=\mathscr{B}$.

The above corollary states that every closed, linear, time-invariant behavior admits a pencil representation. Moreover, as shown in $\left[19\right.$, Thm. 9], the spaces $\mathscr{B} / \mathscr{B}^{1}$ and $\mathscr{B} / \mathscr{B}^{0}$ that appear in the representation $\mathscr{B}_{\mathrm{p}}\left(\mathscr{B} / \mathscr{B}^{1}, \mathscr{B} / \mathscr{B}^{0}, W ; M_{1}, M_{0}, M_{2}\right)$ are finite-dimensional. For completeness, we will offer a proof of this fact which we think is more straightforward than the two proofs that were already given for essentially the same fact in [19]. Some notation will be needed. Let $[w]_{k}$ denote the $k$-truncation of an element $w$ of $W^{\mathbb{Z}_{+}}$; if

$$
w=\left(w_{0}, w_{1}, \cdots, w_{k}, w_{k+1}, \cdots\right),
$$

then

$$
[w]_{k}=\left(w_{0}, w_{1}, \cdots, w_{k}\right) .
$$

For subspaces $\mathscr{B}$ of $W^{\mathbb{Z}_{+}}$, write

$$
\mathscr{B}_{k}=\left\{[w]_{k} \mid w \in \mathscr{B}\right\} .
$$

Define a sequence of subspaces of $W$ by

$$
W_{k}^{0}(\mathscr{B})=\left\{w \in W \mid(0,0, \cdots, 0, w) \in \mathscr{B}_{k}\right\} .
$$

We shall let $\mathscr{B}$ be a fixed linear time-invariant behavior, and write $W_{k}^{0}$ rather than $W_{k}^{0}(\mathscr{B})$. It is immediate from $\sigma \mathscr{B} \subset \mathscr{B}$ that $W_{k+1}^{0} \subset W_{k}^{0}$ for all $k$. Because $W$ is finite-dimensional, the sequence of subspaces $W_{0}^{0} \supset W_{1}^{0} \supset \cdots$ must reach a limit after a finite number of steps; the limit subspace will be denoted by $W^{0}$. We now prove the following lemma.

LEMMA 2.4. Suppose that $\mathscr{B}$ is closed. Let $k_{0}$ be such that $W_{k_{0}}^{0}=W^{0}$, and let $\Phi: \mathscr{B} \rightarrow \mathscr{B}_{k_{0}}$ denote the mapping $w \mapsto[w]_{k_{0}}$. Under these conditions, we have

$$
\operatorname{ker} \Phi \subset \mathscr{B}^{0} \text {. }
$$

Proof. Since $\mathscr{B}^{0}$ is by definition the largest $\sigma^{*}$-invariant subspace of $\mathscr{B}$, it suffices to show that $\operatorname{ker} \Phi$ is $\sigma^{*}$-invariant. Take $w \in \operatorname{ker} \Phi$; we want to show that also $\sigma^{*} w \in$ $\operatorname{ker} \Phi$, which will follow if we can prove that $\sigma^{*} w \in \mathscr{B}$. For this, it is sufficient to show that

$$
\left[\sigma^{*} w\right]_{j} \in \mathscr{B}_{j} \quad \forall j \geqq 0,
$$

by the closedness of $\mathscr{B}$. For $0 \leqq j \leqq k_{0}+1,\left[\sigma^{*} w\right]_{j}=0$ and so the condition $(2.25)$ is certainly satisfied. To proceed by induction, suppose that $\left[\sigma^{*} w\right]_{i} \in \mathscr{B}_{i}$ for some $i \geqq$ $k_{0}+1$. Let $\tilde{w} \in \mathscr{B}$ be such that $[\tilde{w}]_{i}=\left[\sigma^{*} w\right]_{i}$. We then have $[w-\sigma \tilde{w}]_{i-1}=0$, and therefore,

$$
w_{i}-\tilde{w}_{i+1} \in W_{i}^{0}=W_{i+1}^{0} .
$$

From (2.26) and the fact that $\left[\sigma^{*} w-\tilde{w}\right]_{i}=0$, it follows that

$$
\left[\sigma^{*} w-\tilde{w}\right]_{i+1} \in \mathscr{B}_{i+1} \text {. }
$$


Since $[\tilde{w}]_{i+1}$ obviously belongs to $\mathscr{B}_{i+1}$, we may conclude that $\left[\sigma^{*} w\right]_{i+1} \in \mathscr{B}_{i+1}$, which is what we wanted to prove.

Remark 2.5. From the lemma, we easily derive that $W^{0}$, the limit of the sequence in (2.23), is equal to $\chi \mathscr{B}^{\circ}$.

PROPOSITION 2.6. If a linear, time-invariant behavior $\mathscr{B}$ is closed, then $\mathscr{B} / \mathscr{B}^{0}$ is finite-dimensional.

Proof. By the lemma, we have

$$
\operatorname{dim} \mathscr{B} / \mathscr{B}^{0} \leqq \operatorname{dim} \mathscr{B} / \operatorname{ker} \Phi=\operatorname{dim} \operatorname{im} \Phi \leqq \operatorname{dim} W^{k_{0}+1}=q\left(k_{0}+1\right) .
$$

It is not hard to show directly that the pencil representation obtained above is, in fact, minimal.

Lemma 2.7. If $(Z, X, W ; F, G, H)$ is a pencil representation of the linear, time-invariant behavior $\mathscr{B}$, then

$$
\operatorname{dim} X \geqq \operatorname{dim} \mathscr{B} / \mathscr{B}^{0}
$$

and

$$
\operatorname{dim} Z \geqq \operatorname{dim} \mathscr{B} / \mathscr{B}^{1} .
$$

Proof. Introduce the behavior of the auxiliary variables

$$
\mathscr{Z}=\left\{z: \mathbb{Z}_{+} \mapsto Z \mid \sigma G z=F z\right\} .
$$

By definition of a pencil representation, we have

$$
H \mathscr{Z}=\mathscr{B} .
$$

In analogy with $\mathscr{B}^{0}$, we also introduce

$$
\mathscr{Z}^{0}=\left\{z \in \mathscr{Z} \mid\left(\sigma^{*}\right)^{k} z \in \mathscr{Z} \forall k \geqq 0\right\} .
$$

Obviously, we have

$$
H \mathscr{Z}^{0} \subset \mathscr{B}^{0} .
$$

It is easily verified that, in fact,

$$
\mathscr{Z}^{0}=\left\{z \in \mathscr{Z} \mid G z_{0}=0\right\},
$$

which shows that $\mathscr{Z}^{0}$ is the kernel of the mapping which assigns the element $G z_{0}$ of $X$ to a given $z \in \mathscr{Z}$. As a consequence, we get

$$
\operatorname{dim}\left(\mathscr{Z} / \mathscr{L}^{0}\right) \leqq \operatorname{dim} X .
$$

Because of (2.34), we can unambiguously define a mapping $\Psi: \mathscr{Z} / \mathscr{Z}^{0} \rightarrow \mathscr{B} / \mathscr{B}^{0}$ by

$$
\Psi: z \bmod \mathscr{Z}^{0} \mapsto H z \bmod \mathscr{B}^{0} .
$$

Moreover, (2.32) shows that this map is surjective. Therefore,

$$
\operatorname{dim} \mathscr{B} / \mathscr{B}^{0} \leqq \operatorname{dim} \mathscr{Z} / \mathscr{Z}^{0} \leqq \operatorname{dim} X .
$$


For the proof of the second inequality, we introduce

$$
\mathscr{Z}^{1}=\left\{z \in \mathscr{Z}^{0} \mid z_{0}=0\right\}=\left\{z \in \mathscr{Z} \mid z_{0}=0\right\}
$$

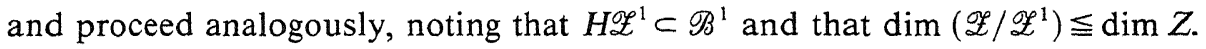

We summarize the main results in the following theorem.

THEOREM 2.8. Let $\mathscr{B}$ be a closed, linear, time-invariant, discrete-time behavior. Then a finite-dimensional minimal pencil representation of $\mathscr{B}$ is given by $\left(\mathscr{B} / \mathscr{B}^{1}, \mathscr{B} / \mathscr{B}{ }^{0}, W\right.$; $\left.M_{1}, M_{0}, M_{2}\right)$, where $\mathscr{B}^{0}$ and $\mathscr{B}^{1}$ are defined by (2.4) and (2.5), respectively, and the mappings $M_{0}, M_{1}$, and $M_{2}$ are defined by requiring that Fig. 1 commutes.

A behavior $\mathscr{B}$ will rarely be given "as such," and consequently the construction of a pencil representation as given above is mainly of theoretical value. Two important ways of prescribing a behavior are the following:

- by data: $\mathscr{B}$ is determined as the smallest closed, linear, shift-invariant subspace of $W^{\mathbb{Z}}$ that contains a given (finite) set of trajectories. This leads to realization procedures involving generalizations of the Hankel matrix: see [20] and, for the case of approximate modeling, [21].

- by equations: $\mathscr{B}$ is determined as the set of all trajectories that satisfy a certain set of differential or difference equations. For the purpose of describing a closed, linear, time-invariant behavior, such equations may always be rewritten in the form $R(\sigma) w=0$, where $R(s)$ is a polynomial matrix [18, Prop. 3.3].

We shall be concerned with the second option in this paper. In the next section, we shall consider systems given by a set of equations $R(\sigma) w=0$, and we shall construct a pencil representation by expressing the spaces $\mathscr{B} / \mathscr{B}^{\circ}$, etc. in terms of the polynomial matrix $R(s)$.

3. Pencil representations from autoregressive equations: discrete time. Let a behavior be given by

$$
R(\sigma) w=0
$$

where $R(s)$ is a polynomial matrix of size $k \times q$, and $\sigma$ denotes the shift. We shall continue to work in discrete time in order to employ the results of the previous section to give a representation in pencil form for the behavior described by (3.1). Similar results can be obtained for systems in continuous time, but these require a different proof technique and will be handled in the next section.

It will be convenient to use an alternative notation for time series, one that is more adapted to the description in terms of a polynomial matrix. Via the correspondence

$$
\left(w_{0}, w_{1}, \cdots\right) \leftrightarrow w_{0} \lambda^{-1}+w_{1} \lambda^{-2}+\cdots,
$$

we can identify $W^{\mathbb{Z}_{+}}$with the set of formal power series (with vanishing constant term) in the parameter $\lambda^{-1}$. This set, to be denoted by $\Omega W$, is a subset of the set $\Lambda W$ of formal Laurent series around infinity in $\lambda$, of which a typical element is

$$
w_{-i-1} \lambda^{i}+w_{-i} \lambda^{i-1}+\cdots+w_{-1}+w_{0} \lambda^{-1}+w_{1} \lambda^{-2}+\cdots .
$$

The natural projection of $\Lambda W$ onto $\Omega W$, effected by "deleting the polynomial part," will be denoted by $\pi_{-}$. Elements of $\Omega W$ will be written as $w(\lambda)$ or sometimes also simply as $w$. 
The action of the shift $\sigma$ on $W^{\mathbb{Z}_{+}}$corresponds on $\Omega W$ to multiplication by $\lambda$ followed by projection:

$$
\sigma w \leftrightarrow \pi_{-}(\lambda w(\lambda)) .
$$

Consequently, the behavior $\mathscr{B}$ given by (3.1) is represented in $\Omega W$ by the set $X^{R}$ that is defined by

$$
X^{R}=\left\{w \in \Omega W \mid \pi_{-}(R(\lambda) w(\lambda))=0\right\} .
$$

The right shift $\sigma^{*}$ is represented in $\Omega W$ by multiplication by $\lambda^{-1}$. Therefore, $\mathscr{B}^{0}$ corresponds to the subspace $N^{R}$ defined by

$$
N^{R}=\left\{w \in \Omega W \mid \pi_{-}\left(\lambda^{-k} R(\lambda) w(\lambda)\right)=0 \forall k \geqq 0\right\}=\{w \in \Omega W \mid R(\lambda) w(\lambda)=0\} .
$$

Finally, $\mathscr{B}^{1}$ is equal to $\sigma^{*} \mathscr{B}^{0}$, which corresponds to $\lambda^{-1} N^{R}$.

The quotient space $\mathscr{B} / \mathscr{B}^{0}$, which plays a role in the pencil representation of the previous section as the space in which the dynamic equation "takes place," is represented as $X^{R} / N^{R}$. We can consider mutiplication by $R(\lambda)$ as a mapping from $X^{R}$ to $\mathbb{R}^{k}[\lambda]$, the set of polynomials with coefficients in $\mathbb{R}^{k}$. The space $N^{R}$ is then precisely the kernel of this mapping, which suggests replacing the quotient space $X^{R} / N^{R}$ by the isomorphic space

$$
X_{R}=\left\{p(\lambda) \in \mathbb{R}^{k}[\lambda] \mid \exists w(\lambda) \in \Omega W \text { s.t. } R(\lambda) w(\lambda)=p(\lambda)\right\} .
$$

The isomorphism is given, of course, by the mapping $M_{R}$ defined as follows:

$$
M_{R}: w(\lambda) \bmod N^{R} \mapsto R(\lambda) w(\lambda) \quad\left(w(\lambda) \in X^{R}\right) .
$$

With some of the notation used in Fig. 1 unchanged, we now introduce the mappings $F, G$, and $H$ by requiring that Fig. 2 below commutes. We then obtain the following theorem.

Theorem 3.1. The behavior given by (3.1) is equal to $\mathscr{B}_{\mathrm{p}}\left(X^{R} / \lambda^{-1} N^{R}, X_{R}, W ; F\right.$, $G, H)$; and this pencil representation is minimal.

Proof. Apart from changes of notation, all we did was replace the representation derived in the previous section by an isomorphic one. The result is therefore immediate from Theorem 2.8 .

Bases for the vector spaces $X_{R}$ and $X^{R} / \lambda^{-1} N^{R}$ may be found by taking $R(s)$ to row reduced form, and then concrete matrix representations for the mappings $F, G$, and $H$ can be obtained. This is worked out in $\S 8$.

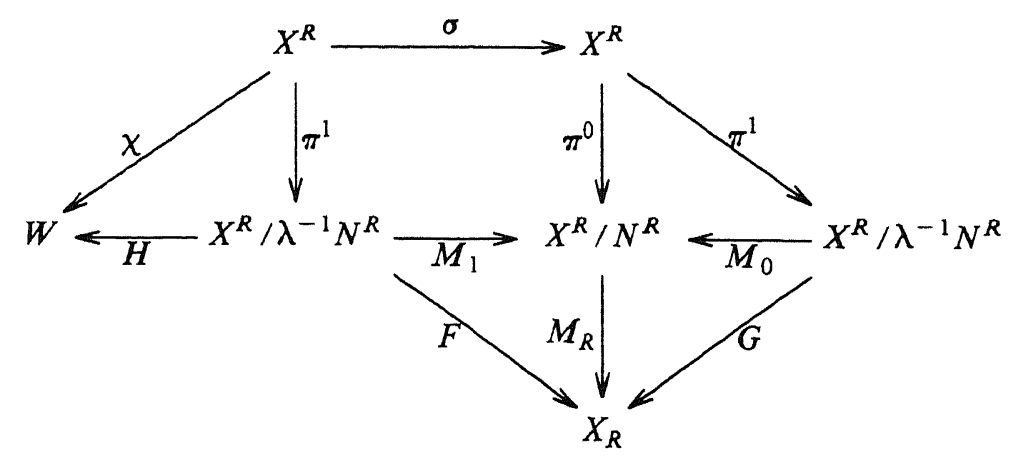

FIG. 2 
4. Pencil representations from autoregressive equations: continuous time. In the discrete-time context, many system properties are conveniently expressed in terms of the behavior itself, and we have used this fact extensively in the previous sections to prove properties of representations; for instance, equivalence between AR and pencil representations could be proved by reducing both to their associated behaviors. For systems in continuous time, however, the representation of a behavior in terms of itself is much less manageable, and we are forced to work with representations in terms of equations. The formal definition of a continuous-time behavior requires the specification of a function class to which the trajectories should belong. We will denote by $\mathscr{F}$ the function class to which the (components of the) trajectories of the external variables belong; the class from which the components of the trajectories of internal (auxiliary) variables are taken will be denoted by $\mathscr{D}$. We will assume that $\mathscr{D}$ is a linear function space that is closed under differentiation and that contains $\mathscr{F}$; differential equations will always be considered in the sense of $\mathscr{D}$. All properties used below will be valid when $\mathscr{F}=\mathscr{D}=C^{\infty}(\mathbb{R})$ (see for instance [15]), but other choices are also possible-however, we shall not go into the axiomatics here. Confer also the discussion in $[3$, Chaps. 4,5$]$. The development below may also be applied to systems in discrete time, although the approach of the preceding two sections would seem to be preferable for its intuitive appeal.

We begin by noting some facts concerning the elimination of auxiliary variables. To interpret the statements in the lemma below, it is useful to remember that with any behavior $\mathscr{B}$ admitting an AR representation we can associate a subspace of the rational vector space $W(\lambda)$ of rational $W$-valued functions in the formal parameter $\lambda$. Indeed, if $R(s)$ is an AR matrix for the given behavior, then $R(\lambda)$ can be viewed as a mapping between rational vector spaces, and its kernel is easily seen to be independent of the choice of the representation. So $\operatorname{ker} R(\lambda)$ is uniquely determined by the behavior. In the interpretation of the previous section, $\operatorname{ker} R(\lambda)$ is just the linear span (over $\mathbb{R}(\lambda)$ ) of the elements of $\mathscr{B}$. In particular, $\operatorname{dim} \operatorname{ker} R(\lambda)$ is the number of inputs in any standard state space description of $\mathscr{B}$.

LEMMA 4.1. Consider a behavior $\mathscr{B}$ given by the equations

$$
\begin{aligned}
& P(\sigma) \xi=0 \\
& w=Q(\sigma) \xi
\end{aligned}
$$

where $P(s)$ and $Q(s)$ are polynomial matrices, and $\xi$ contains auxiliary variables. Denote by $q$ the number of rows of $Q(s)$, by $n$ the number of rows of $P(s)$, and by $r$ the rank of $\left[P^{\top}(s) Q^{\top}(s)\right]^{\top}$. It is always possible to find polynomial matrices $V(s)$ and $R(s)$ such that (i) $V(s)$ has size $(n+q-r) \times n, R(s)$ has size $(n+q-r) \times q$;

(ii) $V(s)$ and $R(s)$ are left coprime, i.e., the matrix $[V(s) R(s)]$ has full row rank for all $s \in \mathbb{C}$;

(iii) $V(s) P(s)+R(s) Q(s)=0$.

If $V(s)$ and $R(s)$ satisfy these properties, then an AR description of the behavior defined by $(4.1)-(4.2)$ is

$$
R(\sigma) w=0
$$

and the following relation holds, where all matrices are interpreted as matrices over the field of rational functions:

$$
\operatorname{ker} R(\lambda)=Q(\lambda)[\operatorname{ker} P(\lambda)]
$$


In particular, we have

$$
\operatorname{dim} \operatorname{ker} R(\lambda)=\operatorname{rank}\left(\begin{array}{c}
P(\lambda) \\
Q(\lambda)
\end{array}\right)-\operatorname{rank} P(\lambda) .
$$

Proof. For instance by reduction to Hermite form [8, p. 375] we can find a unimodular matrix $U(s)$ of size $(n+q) \times(n+q)$ such that

$$
\left(\begin{array}{ll}
U_{11}(s) & U_{12}(s) \\
U_{21}(s) & U_{22}(s)
\end{array}\right)\left(\begin{array}{l}
P(s) \\
Q(s)
\end{array}\right)=\left(\begin{array}{c}
T(s) \\
0
\end{array}\right)
$$

where $T(s)$ has full row rank. Clearly then, the number of rows of $T(s)$ must be equal to $r$, and so the dimensions of $U_{21}(s)$ and $U_{22}(s)$ are $(n+q-r) \times n$ and $(n+q-r) \times q$, respectively. It is easily verified also that conditions (ii) and (iii) above are satisfied by taking $V(s)=U_{21}(s)$ and $R(s)=U_{22}(s)$.

Suppose now that $V(s)$ and $R(s)$ satisfy conditions (i)-(iii). We can then find polynomial matrices $U_{1}(s)$ and $U_{2}(s)$ such that the matrix

$$
\left(\begin{array}{cc}
U_{1}(s) & U_{2}(s) \\
V(s) & R(s)
\end{array}\right)
$$

is unimodular. If we write $T(s)=U_{1}(s) P(s)+U_{2}(s) Q(s)$, then we obviously have

$$
\left(\begin{array}{cc}
U_{1}(s) & U_{2}(s) \\
V(s) & R(s)
\end{array}\right)\left(\begin{array}{l}
P(s) \\
Q(s)
\end{array}\right)=\left(\begin{array}{c}
T(s) \\
0
\end{array}\right) .
$$

Moreover, $T(s)$ must be of full row rank, since its number of rows is equal to the rank of $\left[P^{\top}(s) Q^{\top}(s)\right]^{\top}$. This implies that $R(s)$ is an AR matrix for the behavior given by (4.1)-(4.2) (see [15, Cor. 2.3]). The formula (4.4) is obtained by interpreting (4.7) as a rational matrix equation and using straightforward linear algebra, and (4.5) is an immediate consequence. This completes the proof of the lemma.

In the discrete-time context, we used quotients of sequence spaces to construct the vector spaces that are needed in a pencil representation. It should be noted that the end result would have been the same if we would have replaced the sequence spaces by corresponding spaces of rational vector functions; in particular, the space $W(\lambda)$ of rational functions with values in $W$ may be substituted for $\Lambda W$, and $\lambda^{-1} W\left[\left[\lambda^{-1}\right]\right]$ (the space of strictly proper rational $W$-valued functions) for $\Omega W$. For continuous-time systems, the use of sequence spaces is less natural, and we shall use the rational setting. This will also facilitate comparison with the results of Fuhrmann (see, e.g., [6]). The symbol $\pi_{-}$will be used now for the natural projection of $X(\lambda)$ (where $X$ is any vector space) onto $\lambda^{-1} X\left[\left[\lambda^{-1}\right]\right]$. For an element $w(\lambda)$ of $\lambda^{-1} W\left[\left[\lambda^{-1}\right]\right]$, the value of $s w(s)$ at infinity will be denoted by $w_{-1}$ in accordance with the notation of [6], rather than by $w_{0}$ as would be suggested by (3.2).

The next theorem is the main result of this section. Essentially, it shows how to solve the equations that we obtain by requiring that Fig. 2 commutes.

THEOREM 4.2. Let a system be given in $\operatorname{AR}$ form (1.2), with $R(s) \in \mathbb{R}^{k \times q}(s)$ of full row rank. Consider the following spaces of rational vector functions in a formal parameter $\lambda$ :

$$
\begin{gathered}
X^{R}=\left\{w(\lambda) \in \lambda^{-1} W\left[\left[\lambda^{-1}\right]\right] \mid \pi_{-} R(\lambda) w(\lambda)=0\right\}, \\
X_{R}=\left\{p(\lambda) \in \mathbb{R}^{k}[\lambda] \mid \exists w(\lambda) \in \lambda^{-1} W\left[\left[\lambda^{-1}\right]\right] \text { s.t. } p(\lambda)=R(\lambda) w(\lambda)\right\}, \\
N^{R}=\left\{w(\lambda) \in \lambda^{-1} W\left[\left[\lambda^{-1}\right]\right] \mid R(\lambda) w(\lambda)=0\right\} .
\end{gathered}
$$


The following mappings ( $G$ and $F$ from $X^{R} / \lambda^{-1} N^{R}$ to $X_{R}, H$ from $X^{R} / \lambda^{-1} N^{R}$ to $W$ ) are well defined:

$$
\begin{gathered}
G: w(\lambda) \bmod \lambda^{-1} N^{R} \mapsto R(\lambda) w(\lambda), \\
F: w(\lambda) \bmod \lambda^{-1} N^{R} \mapsto R(\lambda) \pi_{-}(\lambda w(\lambda)), \\
H: w(\lambda) \bmod \lambda^{-1} N^{R_{1}} w_{-1} .
\end{gathered}
$$

With these definitions, $\left(X^{R} / \lambda^{-1} N^{R}, X_{R}, W ; F, G, H\right)$ is a minimal pencil representation of the behavior given by (1.2).

Proof. It is easily verified that the mappings $F, G$, and $H$ are indeed well-defined. Because $\lambda^{-1} N^{R}$ is contained in $N^{R}$, it is obvious from the definition (4.11) that $G$ is surjective. If, for some $w(\lambda) \in X^{R}$, both $w_{-1}=0$ and $R(\lambda) w(\lambda)=0$, then $\lambda w(\lambda)$ belongs to $N^{R}$ so $w(\lambda)$ belongs to $\lambda^{-1} N^{R}$. This shows that the mapping $\left[G^{\top} H^{\top}\right]^{\top}$ is injective. Furthermore, suppose that $s \in \mathbb{C}$ and $w(\lambda) \in X^{R}$ are such that we have

$$
\begin{gathered}
s R(\lambda) w(\lambda)-R(\lambda) \pi_{-}(\lambda w(\lambda))=0 \\
w_{-1}=0 .
\end{gathered}
$$

Because of (4.15), $\pi_{-}(\lambda w(\lambda))$ is equal to $\lambda w(\lambda)$, and (4.14) may be rewritten as

$$
(s-\lambda) R(\lambda) w(\lambda)=0 .
$$

Of course, this implies that $R(\lambda) w(\lambda)=0$. Because we also have (4.15), it follows that $w(\lambda) \in \lambda^{-1} N^{R}$. By the definitions, this shows that $\left[s G^{\top}-F^{\top} H^{\top}\right]^{\top}$ is injective for all $s \in \mathbb{C}$. By the criterion given in Proposition 1.1, we have now shown that the pencil representation given by $F, G$, and $H$ is minimal.

We still must show that this pencil representation describes the same behavior as the given AR representation. For this purpose, we use the preceding lemma. Let $n$ denote the dimension of $X_{R}$ and write $r$ for the dimension of $X^{R} / \lambda^{-1} N^{R}$; then $r$ is also the rank of $\left[s G^{\top}-F^{\top} H^{\top}\right]^{\top}$, since we have shown that this matrix has full column rank. Because $G$ is surjective and $\operatorname{ker} G=N^{R} / \lambda^{-1} N^{R}$, we can write

$$
r-n=\operatorname{dim} \operatorname{ker} G=\operatorname{dim} N^{R} / \lambda^{-1} N^{R}=\operatorname{dim} \operatorname{ker} R(\lambda)=q-k
$$

since $R(s)$ was assumed to be of full row rank. So, we have $k=n+q-r$, and $R(s)$ has the size required in Lemma 4.1. It remains to find a polynomial matrix $V(s)$ of size $k \times n$ such that conditions (ii) and (iii) of that lemma are satisfied.

We claim that such a polynomial mapping is given by the "evaluation map" which replaces the formal parameter $\lambda$ by the complex number $s$ :

$$
V(s): X_{R} \in p(\lambda) \mapsto p(s) \in \mathbb{C}^{k} .
$$

This map is polynomial because $X_{R}$ consists of polynomial vectors; this is evident when we write a matrix representation of $V(s)$. To verify that condition (ii) holds, we compute, for $w(\lambda) \in X^{R}$ :

$$
\begin{aligned}
V(s)(s G-F) w(\lambda) & =V(s)\left[s R(\lambda) w(\lambda)-R(\lambda)\left(\lambda w(\lambda)-w_{-1}\right)\right] \\
& =s R(s) w(s)-R(s)\left(s w(s)-w_{-1}\right) \\
& =R(s) w_{-1}=R(s) H w(\lambda) .
\end{aligned}
$$

Finally, we must show that $V(s)$ and $R(s)$ are left coprime. For this purpose, it suffices to produce polynomial mappings $Q_{1}(s)$ and $Q_{2}(s)$ such that

$$
V(s) Q_{1}(s)+R(s) Q_{2}(s)=I \text {. }
$$


By assumption, $R(s)$ has full row rank, so it has a rational right inverse, say $T(s)$. We split $T(s)$ into a polynomial and a strictly proper part, denoted, respectively, by $T_{+}(s)$ and $T_{-}(s)$. Obviously, we have

$$
R(s) T_{-}(s)=I-R(s) T_{+}(s)
$$

where the right-hand side is polynomial. It follows that the columns of $R(\lambda) T_{-}(\lambda)$ belong to $X_{R}$. Consequently, there exists a constant matrix $Q_{1}$ such that

$$
R(s) T_{-}(s)=V(s) Q_{1} .
$$

Writing $T_{+}(s)$ as $Q_{2}(s)$, we get

$$
V(s) Q_{1}+R(s) Q_{2}(s)=R(s) T_{-}(s)+R(s) T_{+}(s)=R(s) T(s)=I .
$$

5. Realization with a causal input/output structure. In the realization procedure of the previous section, we could replace the quotient space $X^{R} / N^{R}$ by the space of polynomials $X_{R}$, because we had a natural isomorphism available between these two spaces, given essentially by multiplication by $R(\lambda)$. The other space that we used, $X^{R} / \lambda^{-1} N^{R}$, is isomorphic to the direct sum $X_{R} \oplus W^{0}$, where $W^{0}$ is the subspace of $W$ defined by

$$
W^{0}=\left\{w \in W \mid \exists w(\lambda) \in N^{R} \text { s.t. } w=w_{-1}\right\} .
$$

(In other words, we have $W^{0}=H N^{R}$, in full analogy with the discrete-time case-see Remark 2.5.) Indeed, the following holds:

$$
X^{R} / \lambda^{-1} N^{R} \simeq X^{R} / N^{R} \oplus N^{R} / \lambda^{-1} N^{R} \simeq X_{R} \oplus W^{0} .
$$

Unfortunately, the first isomorphism in the formula above must be established by selecting a complement to $N^{R} / \lambda^{-1} N^{R}$ in $X^{R} / \lambda^{-1} N^{R}$, and so we do not have a natural isomorphism available. This is also reflected in the nonuniqueness of "driving-variable" representations as described in [18, Thm. 7.1]. It should be noted that the space $W^{0}$ itself is canonically given (i.e., it is an invariant under external equivalence), and this space will play an important role below.

Now, suppose that we add more structure by dividing the external variables into inputs and outputs. Such a division is given by a decomposition of the external variable space $W$ as the direct sum of two subspaces $Y$ and $U$, corresponding to a splitting of the defining AR matrix $R(s)$ as

$$
R(s)=\left[R_{1}(s) \quad R_{2}(s)\right] .
$$

The projection onto $U$ along $Y$ will be denoted by $\pi_{U}$, the complementary projection by $\pi_{Y}$. We shall first consider the "causal" situation as described in the following lemma, which is a formalization of remarks in $[18, \S 6]$. General input/output structures will be discussed in the next section.

LEMMA 5.1. With the notations introduced above, the following statements are equivalent:

(i) $R_{1}(s)$ is invertible as a rational matrix, and $R_{1}^{-1}(s) R_{2}(s)$ is proper rational;

(ii) the projection $\pi_{U}$, taken as a mapping from $W^{0}$ to $U$, is an isomorphism;

(iii) there exists a mapping $D: U \mapsto Y$ such that

$$
W^{0}=\left\{\left(\begin{array}{c}
D u \\
u
\end{array}\right) \mid u \in U\right\}
$$

where the vector notation is adapted to the decomposition of $W$ as $Y \oplus U$; 
(iv) $Y$ is a complement of $W^{0}$ in $W$.

Proof. The equivalence between statements (ii), (iii), and (iv) is a matter of straightforward linear algebra. To prove that (i) implies (iii), define

$$
D=\left[-R_{1}^{-1}(s) R_{2}(s)\right]_{s=\infty} \text {. }
$$

Take $w \in W^{0}$, and let $w(\lambda) \in N^{R}$ be such that $w_{-1}=w$. From $R(\lambda) w(\lambda)=0$, we have

$$
\pi_{Y} w(\lambda)+R_{1}^{-1}(\lambda) R_{2}(\lambda) \pi_{U} w(\lambda)=0,
$$

and this implies

$$
\pi_{Y} w_{-1}=D \pi_{U} w_{-1} .
$$

Conversely, suppose that $w \in W$ is of the form

$$
w=\left(\begin{array}{c}
D u \\
u
\end{array}\right) \text {. }
$$

Define $w(\lambda)$ by

$$
w(\lambda)=\lambda^{-1}\left(\begin{array}{c}
R_{1}^{-1}(\lambda) R_{2}(\lambda) u \\
u
\end{array}\right)
$$

then $w(\lambda) \in N^{R}$ and $w_{-1}=w$, so that $w \in W^{0}$.

Now, assume that (ii)-(iv) hold. Let $N(\lambda)$ be a basis matrix for the rational vector space $\operatorname{ker} R(\lambda)$; we may assume that $N(\lambda)$ is proper rational, and that its leading coefficient matrix $N_{0}=[N(s)]_{s=\infty}$ has full column rank. (To see this, note that by reducing $R(\lambda)$ to row reduced form one actually writes $R(\lambda)=[S(\lambda) 0] B(\lambda)$ where $S(\lambda)$ is a nonsingular polynomial matrix, and $B(\lambda)$ is bicausal. One may then take $N(\lambda)=B^{-1}(\lambda)\left[\begin{array}{ll}0 & I\end{array}\right]^{\top}$.) Under these conditions, $N_{0}$ is a basis matrix for $W^{0}$ and it follows that $\operatorname{dim} U=\operatorname{dim} W^{0}=q-k$ where $k$ is the number of rows of $R(\lambda)$. So, $\operatorname{dim} Y=k$ and it is seen that the matrix $R_{1}(\lambda)$ is square. To prove that $R_{1}(\lambda)$ is invertible, suppose that $R_{1}(\lambda) y(\lambda)=0$ for some $y(\lambda) \in Y(\lambda)$ not equal to zero. It is no restriction of the generality to assume that $y(\lambda)$ is strictly proper with a nonzero leading term $y_{-1}$; but then the vector $\left[\begin{array}{ll}y_{-1}^{\top} & 0\end{array}\right]^{\top}$ belongs to $Y \cap W^{0}$ and so should be zero according to (iv). Finally, note that by definition we have

$$
R_{1}(\lambda) \pi_{Y} N(\lambda)+R_{2}(\lambda) \pi_{U} N(\lambda)=0
$$

Moreover, the rational matrix $\pi_{U} N(\lambda)$ is proper with an invertible leading coefficient matrix, as is seen from (ii), and this implies that

$$
R_{1}^{-1}(\lambda) R_{2}(\lambda)=-\pi_{Y} N(\lambda)\left(\pi_{U} N(\lambda)\right)^{-1}
$$

is proper rational. This completes the proof of the lemma.

In the remainder of this section, we assume that $R_{1}(s)$ in (5.3) is invertible, and that $R_{1}^{-1}(s) R_{2}(s)$ is proper rational. To construct the parameters in a standard statespace representation of the behavior given by $R(s)$, define a mapping $\Phi$ from $X^{R} / \lambda^{-1} N^{R}$ to $X_{R} \oplus U$ by

$$
\Phi: w(\lambda) \bmod \lambda^{-1} N^{R} \mapsto\left(\begin{array}{c}
R(\lambda) w(\lambda) \\
\pi_{U} w_{-1}
\end{array}\right)
$$

(it is easily seen that this is well-defined). To prove that $\Phi$ is injective, let $w(\lambda) \in X^{R}$ be such that $R(\lambda) w(\lambda)=0$ and $\pi_{U} w_{-1}=0$. For such a $w(\lambda)$, we get $w(\lambda) \in N^{R}$ so $w_{-1} \in W^{0}$. The condition $\pi_{U} w_{-1}=0$ implies $w_{-1} \in Y$, so that $w_{-1} \in Y \cap W^{0}=\{0\}$, which proves that $w(\lambda) \in \lambda^{-1} N^{R}$. This shows that $\Phi$ is injective; the fact that $\Phi$ is actually 
an isomorphism then follows easily by a dimension argument. Using the obvious facts $\left[\begin{array}{ll}I & 0\end{array}\right] \Phi=G$ and $\left[\begin{array}{ll}0 & I\end{array}\right] \Phi=\pi_{U} H$, we can now write down the diagram below which we use to define the mappings $A, B, C$, and $D$ that will appear in an input/state/output representation of the given behavior.

We can give more explicit expressions for the four mappings defined by requiring that Fig. 3 commutes. Note that $R_{1}^{-1}(s) p(s)$ is strictly proper if $p(\lambda) \in X_{R}$; indeed, suppose that $p(s)=R_{1}(s) \pi_{Y} w(s)+R_{2}(s) \pi_{U} w(s)$ for $w(\lambda) \in X^{R}$, then

$$
R_{1}^{-1}(s) p(s)=\pi_{Y} w(s)+R_{1}^{-1}(s) R_{2}(s) \pi_{U}(s) w(s)
$$

and this is obviously strictly proper. With this information, it is easily seen that the inverse of the isomorphism $\Phi$ may be given as follows:

$$
\Phi^{-1}: X_{R} \oplus U \ni\left(\begin{array}{c}
p(\lambda) \\
u
\end{array}\right) \mapsto\left(\begin{array}{c}
R_{1}^{-1}(\lambda) p(\lambda)-\lambda^{-1} R_{1}^{-1}(\lambda) R_{2}(\lambda) u \\
\lambda^{-1} u
\end{array}\right) \bmod \lambda^{-1} N^{R} .
$$

The mapping $\left[\begin{array}{ll}A & B\end{array}\right]$ can now be computed as $M_{R} M_{1} \Phi^{-1}$. Explicitly, this gives:

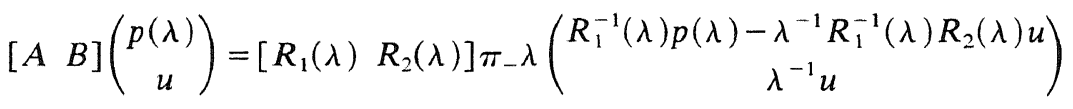

$$
\begin{aligned}
& =R_{1}(\lambda) \pi_{-} \lambda R_{1}^{-1}(\lambda)\left(p(\lambda)-R_{2}(\lambda) u\right)+R_{2}(\lambda) \pi_{-} u \\
& =\pi_{R_{1}} \lambda p(\lambda)-\pi_{R_{1}} R_{2}(\lambda) u,
\end{aligned}
$$

where the notation $\pi_{R_{1}}$ is used, following [6], for the projection on $X_{R}$ given by

$$
\pi_{R_{1}}: p(\lambda) \mapsto R_{1}(\lambda) \pi_{-} R_{1}^{-1}(\lambda) p(\lambda) .
$$

In particular, we find

$$
A: p(\lambda) \mapsto \pi_{R_{1}} \lambda p(\lambda)=\lambda p(\lambda)-R_{1}(\lambda)\left[R_{1}^{-1}(\lambda) p(\lambda)\right]_{-1}
$$

and

$$
B: u \mapsto-\pi_{R_{1}} R_{2}(\lambda) u .
$$

The expression for $B$ may also be written in a different way if we introduce a constant matrix $D_{\infty}$ by

$$
D_{\infty}=\left[R_{1}^{-1}(s) R_{2}(s)\right]_{s=\infty} ;
$$

namely,

$$
B: u \mapsto-R_{2}(\lambda) u+R_{1}(\lambda) D_{\infty} u .
$$

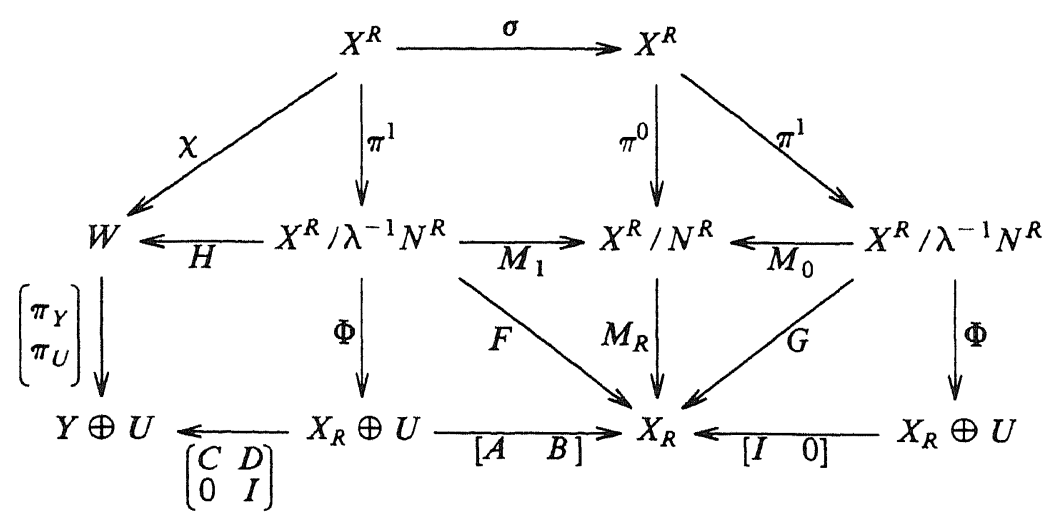

FIG. 3 
Quite similarly, we obtain explicit expressions for the mappings $C$ and $D$ from the formula $\left[\begin{array}{ll}C & D\end{array}\right]=\pi_{Y} H \Phi^{-1}$. We find

$$
C: p(\lambda) \mapsto\left[R_{1}^{-1}(\lambda) p(\lambda)\right]_{-1}
$$

and

$$
D: u \mapsto-D_{\infty} u .
$$

So, in this way we recover Fuhrmann's realization of a transfer matrix $-R_{1}^{-1}(s) R_{2}(s)$ in left matrix fractional representation. Notice that actually we proved more: it is known from Fuhrmann's work that the realization is minimal under transfer equivalence if and only if the fractional representation is coprime, whereas we have shown here that the realization is always minimal under external equivalence. The condition for minimality under transfer equivalence can be derived from this.

It is also possible to set up diagrams to define single mappings from the quadruple $(A, B, C, D)$. For instance, by transforming Fig. 3 we obtain Fig. 4, which can be used to define the mapping $A$. This clearly displays $A$ as a version of the shift.

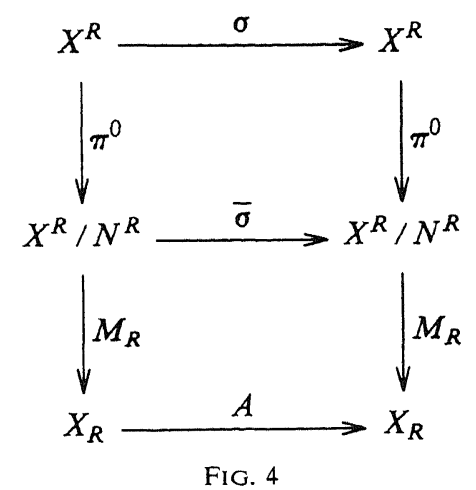

6. Realization with a general input/output structure. In the case where we have given a not necessarily causal input/output description, our aim is to obtain a representation in descriptor form. To arrive at this representation, it turns out to be advantageous to use the pencil form as an intermediate step; the descriptor form can be derived from the pencil form in a straightforward way, as will now be shown.

Let a pencil representation $(Z, X, W ; F, G, H)$ be given, along with a decomposition $W=Y \oplus U$ and associated projections $\pi_{Y}$ and $\pi_{U}$. Decompose the internal variable space $Z$ as $Z_{0} \oplus Z_{1} \oplus Z_{2}$ where $Z_{1}=\operatorname{ker} G \cap \operatorname{ker} \pi_{U} H$, and $Z_{1} \oplus Z_{2}=\operatorname{ker} G$. Accordingly, write

$$
\begin{gathered}
G=\left[\begin{array}{lll}
G_{0} & 0 & 0
\end{array}\right], \quad F=\left[\begin{array}{lll}
F_{0} & F_{1} & F_{2}
\end{array}\right], \\
\pi_{Y} H=\left[\begin{array}{lll}
H_{00} & H_{01} & H_{02}
\end{array}\right], \quad \pi_{U} H=\left[\begin{array}{lll}
H_{u 0} & 0 & H_{u 2}
\end{array}\right] .
\end{gathered}
$$

The matrix $H_{u 2}$ has full column rank, and by renumbering the $u$-variables if necessary, we can write

$$
H_{u 0}=\left(\begin{array}{l}
H_{10} \\
H_{20}
\end{array}\right), \quad H_{u 2}=\left(\begin{array}{l}
H_{12} \\
H_{22}
\end{array}\right)
$$


where $H_{22}$ is invertible (or empty, if $\operatorname{ker} G \subset \operatorname{ker} \pi_{U} H$ ). The system equations take the form (in obvious notation):

$$
\begin{aligned}
& \sigma G_{0} z_{0}=F_{0} z_{0}+F_{1} z_{1}+F_{2} z_{2}, \\
& y=H_{00} z_{0}+H_{01} z_{1}+H_{02} z_{2}, \\
& u_{1}=H_{10} z_{0}+H_{12} z_{2}, \\
& u_{2}=H_{20} z_{0}+H_{22} z_{2} .
\end{aligned}
$$

We can now solve for $z_{2}$ and obtain a description in descriptor form

$$
\begin{aligned}
& \sigma E z=A z+B u, \\
& y=C z+D u
\end{aligned}
$$

where the parameters are defined as follows:

$$
\begin{gathered}
E=\left(\begin{array}{cc}
G_{0} & 0 \\
0 & 0
\end{array}\right), \quad A=\left(\begin{array}{cc}
F_{0}-F_{2} H_{22}^{-1} H_{20} & F_{1} \\
H_{10}-H_{12} H_{22}^{-1} H_{20} & 0
\end{array}\right), \quad B=\left(\begin{array}{cc}
0 & F_{2} H_{22}^{-1} \\
-I & H_{12} H_{22}^{-1}
\end{array}\right), \\
C=\left[\begin{array}{lll}
H_{00}-H_{02} H_{22}^{-1} H_{20} & H_{01}
\end{array}\right], \quad D=\left[\begin{array}{ll}
0 & H_{02} H_{22}^{-1}
\end{array}\right] .
\end{gathered}
$$

Remark 6.1. The essence of the above construction is that as many $z$-variables as possible are replaced by $u$-variables. If this is not considered important, then, of course a simpler construction is possible: just write

$$
\begin{aligned}
& \sigma\left(\begin{array}{c}
G \\
0
\end{array}\right) z=\left(\begin{array}{c}
F \\
\pi_{U} H
\end{array}\right) z+\left(\begin{array}{c}
0 \\
-I
\end{array}\right) u, \\
& y=\pi_{Y} H z .
\end{aligned}
$$

This simple solution will in general produce a nonminimal descriptor representation even if one starts with a minimal pencil representation. The more elaborate construction above behaves nicely with respect to minimality properties, as shown below and as further detailed in [9].

The following lemma, which will be needed below, also sheds some light on the role of the $u_{2}$-variables. Recall that, in the construction above, these variables serve to parametrize the subspace $\pi_{U} H[\operatorname{ker} G]$ of $W$.

LEMMA 6.2. Consider a pencil representation (1.5) and an equivalent AR representation (1.2); assume that $G$ is surjective and that $\left[G^{\top} H^{\top}\right]^{\top}$ is injective. Let the subspace $W^{0}$ of $W$ be defined by (5.1). We then have

$$
W^{0}=H[\operatorname{ker} G] .
$$

Proof. It follows from Lemma 4.1 that a rational vector $w(\lambda)$ belongs to $\operatorname{ker} R(\lambda)$ if and only if there exists a rational vector $z(\lambda)$ such that

$$
\left(\begin{array}{c}
0 \\
w(\lambda)
\end{array}\right)=\left(\begin{array}{c}
\lambda G-F \\
H
\end{array}\right) z(\lambda)
$$

Now assume that $w(\lambda)$ is strictly proper; because $\left[G^{\top} H^{\top}\right]^{\top}$ is injective, it then follows that $z(\lambda)$ is also strictly proper, and that its leading coefficient $z_{-1}$ satisfies $G z_{-1}=0$. Moreover, we have $w_{-1}=H z_{-1}$. If follows that $W^{0} \subset H[\operatorname{ker} G]$. Now, it has already been shown in the proof of Lemma 5.1 that $\operatorname{dim} W^{0}=\operatorname{dim} \operatorname{ker} R(\lambda)$. Moreover, using (4.5) and the assumptions, we obtain

(6.15) $\operatorname{dim} \operatorname{ker} R(\lambda)=\operatorname{rank}\left(\begin{array}{c}\lambda G-F \\ H\end{array}\right)-\operatorname{rank}(\lambda G-F)=\operatorname{dim} \operatorname{ker}(\lambda G-F)=\operatorname{dim} \operatorname{ker} G$ so that $\operatorname{dim} \operatorname{ker} G=\operatorname{dim} W^{0}$. Since $\operatorname{dim} \operatorname{ker} G=\operatorname{dim} H[\operatorname{ker} G]$ because $\left[G^{\top} H^{\top}\right]^{\top}$ is injective, this leads to the desired conclusion. 
Note that for minimal pencil representations, this characterization of $W^{0}$ in pencil terms can also be derived from the realization in $\S 4$.

7. Indices and minimality. In this section, we will discuss the minimality of descriptor representations. While for standard state space systems there is only one index that plays a role to determine the minimality (viz., the dimension of the state space), there are three such indices for descriptor systems: the rank of $E$, the column defect of $E$ (dim $\operatorname{ker} E=$ the number of columns minus the rank), and the row defect of $E$ (codim im $E=$ the number of rows minus the rank). A minimal descriptor representation is, by definition, one in which each of these three indices is minimal within the set of descriptor representations for a given behavior. Note that, with this definition, even the existence of a minimal representation is not trivial. Our strategy will be to establish first lower bounds for each of the three indices separately, and to show next that these minima can be achieved simultaneously. The fact that this is possible also shows that, by minimizing the three indices above, one automatically minimizes the number of descriptor variables ( $=$ the number of columns of $E=$ rank + column defect) and the number of equations (= the number of rows of $E=$ rank + row defect).

PROPOSITION 7.1. Let an input/output behavior be given by autoregressive equations

$$
\left[\begin{array}{ll}
R_{1}(\sigma) & R_{2}(\sigma)
\end{array}\right]\left(\begin{array}{l}
y \\
u
\end{array}\right)=0 .
$$

Write $n$ for the sum of the minimal row indices of $R(s)$ (stated in other terms, $n$ is the maximal degree of the full-size minors of $R(s)$ ). Suppose that a descriptor representation of the behavior determined by (7.1) is given by

$$
\begin{aligned}
& \sigma E \xi=A \xi+B u, \\
& y=C \xi+D u .
\end{aligned}
$$

Under these conditions, the rank of $E$ is at least equal to $n$.

Proof. By a suitable choice of coordinates and introduction of new variables, the descriptor equations (7.2)-(7.3) may be written as follows:

$$
\begin{aligned}
& \sigma \xi_{1}=A_{11} \xi_{1}+A_{12} \xi_{2}+B_{1} \eta, \\
& 0=A_{21} \xi_{1}+A_{22} \xi_{2}+B_{2} \eta \\
& \left(\begin{array}{l}
y \\
u
\end{array}\right)=\left(\begin{array}{ll}
C & D \\
0 & I
\end{array}\right)\left(\begin{array}{l}
\xi \\
\eta
\end{array}\right) .
\end{aligned}
$$

The algorithm of [15] may be used to reduce this to state-space (driving-variable) form; the dimension of the state space will be at most equal to the length of the vector $\xi_{1}$, which in turn is equal to the rank of $E$. On the other hand, it is well known (see $[19, \mathrm{Thm} .6])$ that the dimension of the state space must be at least equal to the sum of the minimal row indices of $R(s)$. The stated result follows.

The following two lemmas show that both observability at infinity and reachability at infinity (see for instance [12]) are necessary conditions for minimality of descriptor representations under external equivalence. This is unlike the situation for the finite modes, where minimality under external equivalence requires observability but not controllability [18, Cor. 4.7].

LEMMA 7.2. A necessary condition for (7.2)-(7.3) to be a minimal descriptor representation is that the matrix $\left[E^{\top} C^{\top}\right]^{\top}$ is injective. 
Proof. Suppose that the condition of the lemma is not satisfied, so that ker $E$ and ker $C$ have a nontrivial intersection. By a suitable choice of coordinates, we may then write

$$
E=\left[\begin{array}{ll}
E_{1} & 0
\end{array}\right], \quad C=\left[\begin{array}{ll}
C_{1} & 0
\end{array}\right]
$$

where the number of the columns in the zero matrices is equal to $\operatorname{dim}(\operatorname{ker} E \cap \operatorname{ker} C$ ). The equations (7.2)-(7.3) will then appear in the form

$$
\begin{aligned}
& \sigma E_{1} \xi_{1}=A_{11} \xi_{1}+A_{12} \xi_{2}+B u, \\
& y=C_{1} \xi_{1}+D u .
\end{aligned}
$$

Denote the "equation space" (the space into which $E$ maps) by $X_{\mathrm{e}}$. Let $X_{\mathrm{e}}^{\prime}$ and $T: X_{\mathrm{e}} \rightarrow X_{\mathrm{e}}^{\prime}$ be such that $T$ is surjective and satisfies $\operatorname{ker} T=\operatorname{im} A_{12}$. The equations (7.8)-(7.9) are equivalent to

$$
\begin{aligned}
& \sigma T E_{1} \xi_{1}=T A_{11} \xi_{1}+T B u, \\
& y=C_{1} \xi_{1}+D u .
\end{aligned}
$$

We want to show that this system precedes the original system in the partial ordering determined by the three indices (rank, column defect, row defect) introduced above. That is, we want to show that the following inequalities hold, with strict inequality in at least one case:

$$
\begin{aligned}
& \operatorname{rank} T E_{1} \leqq \operatorname{rank} E \\
& \operatorname{dim} \operatorname{ker} T E_{1} \leqq \operatorname{dim} \operatorname{ker} E \\
& \text { codim im } T E_{1} \leqq \operatorname{codim} \operatorname{im} E
\end{aligned}
$$

As to (7.12), we have

$$
\begin{aligned}
\operatorname{dim} \operatorname{im} T E_{1} & =\operatorname{dim} \operatorname{im} E_{1}-\operatorname{dim}\left(\operatorname{ker} T \cap \operatorname{im} E_{1}\right) \\
& \leqq \operatorname{dim} \operatorname{im} E_{1}=\operatorname{dim} \operatorname{im} E
\end{aligned}
$$

with equality if and only if

$$
\operatorname{im} A_{12} \cap \operatorname{im} E_{1}=\{0\} \text {. }
$$

We next consider (7.13):

$$
\text { dim } \begin{aligned}
\operatorname{der} T E_{1} & =\operatorname{dim} \operatorname{ker} E_{1}+\operatorname{dim}\left(\operatorname{im} E_{1} \cap \operatorname{im} A_{12}\right) \\
& \leqq \operatorname{dim} \operatorname{ker} E_{1}+\operatorname{dim}(\operatorname{ker} E \cap \operatorname{ker} C)=\operatorname{dim} \operatorname{ker} E
\end{aligned}
$$

where we used the fact that the number of columns of $A_{12}$ is equal to $\operatorname{dim}$ (ker $E \cap$ ker $C$ ). Here, equality holds if and only if $A_{12}$ has full column rank and

$$
\operatorname{im} A_{12} \subset \operatorname{im} E_{1} .
$$

Finally, we verify (7.14):

$$
\text { codim im } T E_{1}=\operatorname{codim} T\left[\operatorname{im} E_{1}\right] \leqq \operatorname{codim} \operatorname{im} E_{1}=\operatorname{codim} \operatorname{im} E
$$

with equality if and only if $\operatorname{ker} T \subset \operatorname{im} E_{1}$, that is, if and only if (7.18) holds. (Here we use the following easily verified fact from linear algebra: if $A$ is a surjective mapping from a space $X$ to a space $Y$, and $X_{0}$ is a subspace of $X$, then $\operatorname{codim} A X_{0} \leqq \operatorname{codim} X_{0}$; equality holds if and only if $\operatorname{ker} A \subset X_{0}$.) 
Now, assume that equality would hold in all three cases. The matrix $A_{12}$ should then have full column rank, so that the rank of $A$ should equal the number of columns of $A_{12}$, which in its turn is equal to $\operatorname{dim}(\operatorname{ker} C \cap \operatorname{ker} E)$. On the other hand, it follows from (7.16) and (7.18) that $A_{12}=0$, so that it would follow that $\operatorname{dim}(\operatorname{ker} C \cap \operatorname{ker} E)=0$, which contradicts our assumption that the subspaces $\operatorname{ker} C$ and $\operatorname{ker} E$ intersect nontrivially. This completes the proof.

Lemma 7.3. A necessary condition for (7.2)-(7.3) to be a minimal descriptor representation is that the matrix $\left[\begin{array}{ll}E & B\end{array}\right]$ is surjective.

Proof. The proof is quite similar to the proof of the previous lemma, and we will not work out all details. Suppose that $\left[\begin{array}{ll}E & B\end{array}\right]$ is not surjective; then, by a suitable choice of coordinates, we can write

$$
E=\left(\begin{array}{c}
E_{1} \\
0
\end{array}\right), \quad B=\left(\begin{array}{c}
B_{1} \\
0
\end{array}\right)
$$

where $\left[\begin{array}{ll}E_{1} & B_{1}\end{array}\right]$ is surjective, and the number of zero rows is equal to codim [ $\left.E B\right]$. With this choice of coordinates, the equations (7.2)-(7.3) can be written as follows:

$$
\begin{aligned}
& \sigma E_{1} \xi=A_{1} \xi+B_{1} u, \\
& 0=A_{2} \xi \\
& y=C \xi+D u .
\end{aligned}
$$

Let $S$ be an injective mapping such that $\operatorname{im} S=\operatorname{ker} A_{2}$. The above equations are equivalent to:

$$
\begin{aligned}
& \sigma E_{1} S \tilde{\xi}=A_{1} S \tilde{\xi}+B_{1} u, \\
& y=C S \tilde{\xi}+D u .
\end{aligned}
$$

To prove the lemma, we need to show that the following three inequalities hold, with strict inequality in at least one case:

$$
\begin{aligned}
& \operatorname{codim} \operatorname{im} E_{1} S \leqq \operatorname{codim} \operatorname{im} E, \\
& \operatorname{dim} \operatorname{ker} E_{1} S \leqq \operatorname{dim} \operatorname{ker} E, \\
& \text { rank } E_{1} S \leqq \text { rank } E .
\end{aligned}
$$

This proof can be conducted as above (or the statement can be derived from the one in the previous lemma by duality).

PROPOSITION 7.4. Let (7.2)-(7.3) be a descriptor representation for the behavior described by (7.1), and define $W^{0}$ as in (5.1). Under these conditions, the following inequalities hold:

$$
\begin{aligned}
& \operatorname{dim} \operatorname{ker} E \geqq \operatorname{dim}\left(Y \cap W^{0}\right), \\
& \operatorname{codim} \operatorname{im} E \geqq \operatorname{codim}\left(Y+W^{0}\right) .
\end{aligned}
$$

Proof. It follows from the lemmas we just proved that we may suppose that the matrix $\left[\begin{array}{ll}E^{\top} & C^{\top}\end{array}\right]^{\top}$ is injective and that the matrix $\left[\begin{array}{ll}E & B\end{array}\right]$ is surjective. Note that the descriptor equations (7.2)-(7.3) may also be written in the following form:

$$
\begin{aligned}
& {\left[\begin{array}{cc}
\sigma E-A & -B
\end{array}\right]\left(\begin{array}{l}
\xi \\
\eta
\end{array}\right)=0,} \\
& \left(\begin{array}{l}
y \\
u
\end{array}\right)=\left(\begin{array}{ll}
C & D \\
0 & I
\end{array}\right)\left(\begin{array}{l}
\xi \\
\eta
\end{array}\right) .
\end{aligned}
$$


Take $w \in W^{0}$; then there exists a proper rational $W$-valued function $w(\lambda)$ satisfying $w_{0}=w$ and $R(\lambda) w(\lambda)=0$. By Lemma 4.1 above, there must exist rational vector functions $\xi(\lambda)$ and $\eta(\lambda)$ such that

$$
\begin{gathered}
{\left[\begin{array}{ll}
\lambda E-A & -B
\end{array}\right]\left(\begin{array}{l}
\xi(\lambda) \\
\eta(\lambda)
\end{array}\right)=0} \\
w(\lambda)=\left(\begin{array}{ll}
C & D \\
0 & I
\end{array}\right)\left(\begin{array}{l}
\xi(\lambda) \\
\eta(\lambda)
\end{array}\right) .
\end{gathered}
$$

These equations may also be written as follows:

$$
\left(\begin{array}{c}
\lambda E-A \\
C
\end{array}\right) \xi(\lambda)=\left(\begin{array}{cc}
0 & B \\
I & -D
\end{array}\right) w(\lambda) .
$$

Since the right-hand side in this equation is proper rational and because $\left[E^{\top} C^{\top}\right]^{\top}$ is injective, $\xi(\lambda)$ must also be proper rational. Moreover, the constant term in the power series development of $\xi(\lambda)$ must satisfy $E \xi_{0}=0$. Now, suppose that $w \in Y \cap W^{0}$. Then, again from (7.35), it follows that $w=C \xi_{0}$; so $w \in C[\operatorname{ker} E]$. Therefore,

$$
\operatorname{dim}\left(Y \cap W^{0}\right) \leqq \operatorname{dim} C[\operatorname{ker} E]=\operatorname{dim} \operatorname{ker} E .
$$

For the proof of the second part, we note that it suffices to show that

$$
\{u \in U \mid B u \in \operatorname{im} E\} \subset \pi_{U} W^{0} .
$$

Indeed, we easily verify that codim $\pi_{U} W^{0}$ (with $\pi_{U} W^{0}$ considered as a subspace of $U)$ is equal to codim $\left(Y+W^{0}\right)$, and we can apply the following rule which holds generally for mappings $A$ between vector spaces $X$ and $Y: \operatorname{codim} A^{-1} Y_{0} \leqq \operatorname{codim} Y_{0}$ ( $Y_{0}$ a subspace of $Y$ ). To show (7.37), let $u \in U$ be such that $B u \in \operatorname{im} E$. The desired conclusion will follow if we can exhibit proper rational functions $\xi(\lambda)$ and $u(\lambda)$ such that $u_{0}=u$ and

$$
(\lambda E-A) \xi(\lambda)=B u(\lambda) .
$$

If we define $y(\lambda)=C \xi(\lambda)+D u(\lambda)$, then $y(\lambda)$ is proper rational and

$$
\left(\begin{array}{c}
\lambda E-A \\
C
\end{array}\right) \xi(\lambda)=\left(\begin{array}{cc}
0 & B \\
I & -D
\end{array}\right)\left(\begin{array}{l}
y(\lambda) \\
u(\lambda)
\end{array}\right)
$$

so that

$$
u=\pi_{U}\left(\begin{array}{l}
y_{0} \\
u_{0}
\end{array}\right) \in \pi_{U} W^{0}
$$

Writing $u(\lambda)=u_{0}+\eta(\lambda)$, we see that it will be sufficient to find a strictly proper solution $\left[\xi(\lambda)^{\top} \eta(\lambda)^{\top}\right]^{\top}$ of the equation

$$
\left[\begin{array}{ll}
\lambda E-A & -B
\end{array}\right]\left(\begin{array}{l}
\xi(\lambda) \\
\eta(\lambda)
\end{array}\right)=B u .
$$

Equivalently, we are looking for a proper solution of the same equation with $B u$ replaced by $\lambda B u$. It follows from Theorem 6.3.12 in [8] that such a solution does indeed exist. 
Remark 7.5. Actually, it is not difficult to display an explicit strictly proper solution to (7.41), if we rewrite this equation by a change of variables as

$$
\left(\begin{array}{cccc}
\lambda I-A_{11} & -A_{12} & -B_{11} & -B_{12} \\
-A_{21} & -A_{22} & I & -B_{22}
\end{array}\right)\left(\begin{array}{c}
\xi_{1}(\lambda) \\
\xi_{2}(\lambda) \\
\eta_{1}(\lambda) \\
\eta_{2}(\lambda)
\end{array}\right)=\left(\begin{array}{c}
x_{0} \\
0
\end{array}\right)
$$

(The identity matrix in the $(2,3)$ position is allowed by the assumption that $[E B]$ is surjective.) A strictly proper solution is

$$
\left(\begin{array}{l}
\xi_{1}(\lambda) \\
\xi_{2}(\lambda) \\
\eta_{1}(\lambda) \\
\eta_{2}(\lambda)
\end{array}\right)=\left(\begin{array}{c}
\left(\lambda I-A_{11}-B_{11} A_{21}\right)^{-1} x_{0} \\
0 \\
A_{21}\left(\lambda I-A_{11}-B_{11} A_{21}\right)^{-1} x_{0} \\
0
\end{array}\right)
$$

as can be verified immediately.

THEOREM 7.6. Let an input/output behavior be given by autoregressive equations (7.1). Denote the sum of the minimal indices of $R(s)$ by $n$, and define $W^{0}$ by (5.1). There exists an externally equivalent descriptor representation (7.2)-(7.3) satisfying the following requirements:

$$
\begin{aligned}
& \operatorname{rank} E=n, \\
& \operatorname{dim} \operatorname{ker} E=\operatorname{dim}\left(Y \cap W^{0}\right), \\
& \text { codim im } E=\operatorname{codim}\left(Y+W^{0}\right) .
\end{aligned}
$$

Moreover, a descriptor representation of the behavior given by (7.1) is minimal if and only if the above three equalities hold.

Proof. In view of the previous results in this section, it only remains to show that a descriptor representation satisfying (7.44)-(7.46) exists. We claim that the representation obtained in the previous section satisfies all requirements, supposing that this representation is formed from a minimal pencil representation (see Proposition 1.1). Using the notation of $\S 6$, we have indeed:

$$
\begin{aligned}
\operatorname{rank} E & =\operatorname{dim} Z_{0}=\operatorname{dim} i m G=\operatorname{dim} X_{R}=n \\
Y \cap W^{0} & =\operatorname{ker} \pi_{U} \cap H[\operatorname{ker} G] \\
& =\left\{w \in W \mid \exists z \in Z: G z=0, w=H z, \pi_{U} w=0\right\} \\
& =H\left[\operatorname{ker} G \cap \operatorname{ker} \pi_{U} H\right]=H Z_{1}
\end{aligned}
$$

(7.49) $\operatorname{dim} \operatorname{ker} E=\operatorname{dim} Z_{1}=\operatorname{dim}\left(\operatorname{ker} \pi_{U} H \cap \operatorname{ker} G\right)=\operatorname{dim}\left(Y \cap W^{0}\right)$

(because $\operatorname{ker} G \cap \operatorname{ker} H=\{0\}$, so that the restriction of $H$ to $Z_{1}$ is injective), and

$$
\operatorname{codim} \operatorname{im} E=\operatorname{dim} U_{1}=\operatorname{codim} \pi_{U} W^{0}=\operatorname{codim}\left(Y+W^{0}\right) .
$$

Remark 7.7. By unimodular operations, we can take the given polynomial matrix $R(s)$ to row proper form (see [8, p. 386]); so, we may assume that $R(s)$ is row proper to start with. This means that we can write

$$
R(s)=\Delta(s) B(s),
$$

where $B(s)$ is right bicausal, and

$$
\Delta(s)=\operatorname{diag}\left(s^{\kappa_{1}}, \cdots, s^{\kappa_{k}}\right) .
$$

It is not difficult to verify that the subspace $W^{0}$ is characterized in these terms as

$$
W^{0}=\operatorname{ker} B(\infty) \text {. }
$$


Note that $B(\infty)$ is nothing but the "leading row coefficient matrix" of $R(s)$. The partitioning of $R(s)$ as $\left[R_{1}(s) R_{2}(s)\right]$ induces a similar partitioning of $B(\infty)$ :

$$
B(\infty)=\left[\begin{array}{ll}
B_{1}(\infty) & B_{2}(\infty)
\end{array}\right]
$$

Using standard manipulations, we find the following expressions for $\operatorname{dim}\left(Y \cap W^{0}\right)$ and $\operatorname{codim}\left(Y+W^{0}\right)$ :

$$
\begin{aligned}
& \operatorname{dim}\left(Y \cap W^{0}\right)=\operatorname{dim} \operatorname{ker} B_{1}(\infty) \\
& \operatorname{codim}\left(Y+W^{0}\right)=\operatorname{codim} i m B_{1}(\infty) .
\end{aligned}
$$

So, we have easy criteria for minimality of descriptor representations of a behavior given by a row proper AR matrix: the rank of $E$ should be equal to the sum of the row indices, and the row and column defects of $E$ should be equal to the corresponding indices of $B_{1}(\infty)$. It also follows that $E$ in a minimal descriptor representation will be square if and only if $B_{1}(\infty)$ is square; this happens if and only if $R_{1}(s)$ is square, that is if the number of $y$-variables is equal to the number of independent equations in an AR representation.

8. Computation. In this section, we will show how to obtain concrete matrix representations in pencil form and in descriptor form, starting from autoregressive equations determined by a $k \times q$ polynomial matrix $R(s)$ of full row rank. For this purpose, we shall construct specific bases for the spaces that appear in the abstract realization of $\S 4$. In the procedure below, the transformation from pencil to descriptor form is not a straightforward implementation of the abstract procedure given in $\S 6$; one reason for this is that, in the abstract version, the crucial subspace $W^{0}$ appears as the image of a certain mapping, whereas in the computation below it appears as a kernel. This leads to a different (dual) method of selecting the $u_{2}$-variables.

The first step is to take the given polynomial matrix $R(s)$ to row proper form $[8$, p. 386]. To alleviate the notation, the resulting equivalent AR matrix will still be denoted by $R(s)$. So we have

$$
R(s)=\Delta(s) B(s)
$$

where $B(s)$ is right bicausal, and

$$
\Delta(s)=\operatorname{diag}\left(s^{\kappa_{1}}, \cdots, s^{\kappa_{k}}\right) .
$$

Now, let $\tilde{B}(s)$ be any matrix such that $\hat{B}(s)=\left[B^{\top}(s) \tilde{B}^{\top}(s)\right]^{\top}$ is bicausal. It will be discussed later how to make a suitable choice for $\tilde{B}(s)$. We can write $R(s)=$ $[\Delta(s) 0] \hat{B}(s)$, and it is seen from this that a basis for $X^{R} / \lambda^{-1} N^{R}$ is given by the equivalence classes modulo $\lambda^{-1} N^{R}$ of the columns of the following matrix of size $q \times(n+q-k)$ :

$$
\hat{B}^{-1}(\lambda)\left[\begin{array}{lllllll}
\lambda^{-1} \cdots \lambda^{-\kappa_{1}} & & & & & & \\
& & \cdots & & & & \\
& & \lambda^{-1} \cdots \lambda^{-\kappa_{k}} & & \\
& & & & \lambda^{-1} & \\
& & & & & \ddots & \\
& & & & & & \lambda^{-1}
\end{array}\right] .
$$

A basis matrix for $X_{R}$ is given by the following matrix of size $k \times n$ :

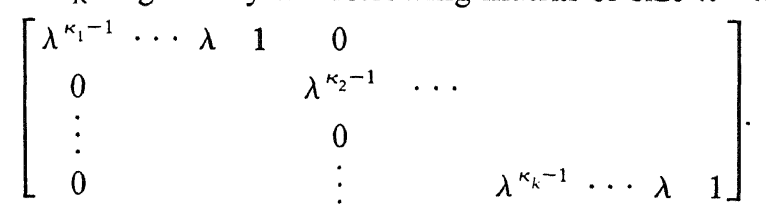


With respect to these bases, we now compute the matrix forms of $F, G$, and $H$. It is easily seen that $G$ will take the form $\left[\begin{array}{ll}I & 0\end{array}\right]$. Because $\hat{B}(\lambda)$ is bicausal, the matrix of $H$ will have the form

$$
H=\hat{B}(\infty)^{-1}\left[\begin{array}{llllllllll}
1 \cdots & \cdots & & & & & & & \\
& & \cdots & & & & & & \\
& & & 1 & \cdots & 0 & & & \\
& & & & & & 1 & & \\
& & & & & & \ddots & \\
& & & & & & & 1
\end{array}\right] .
$$

Here, we see that we will need the inverse of $\hat{B}(\infty)$. Finally, if we let $G(\lambda)$ denote the matrix whose columns are the images under $G$ in $\mathbb{B}^{k}[\lambda]$ of the basis elements for $X^{R} / \lambda^{-1} N^{R}$ displayed above, then we can compute a similar matrix for $F$ by the formula

$$
F(\lambda)=\lambda G(\lambda)-R(\lambda) H
$$

which follows from the definitions of $F, G$, and $H$. This is easily transformed into a matrix expression for $F$ because of the simple basis we chose for $X_{R}$.

Example 8.1. Let $R(s)$ be given by

$$
R(s)=\left(\begin{array}{ccc}
s^{2} & s^{2}+1 & 0 \\
1 & s+2 & 3
\end{array}\right)
$$

The leading row coefficient matrix of this is

$$
B(\infty)=\left(\begin{array}{lll}
1 & 1 & 0 \\
0 & 1 & 0
\end{array}\right)
$$

which has full row rank, so $R(s)$ is already row proper. The row degrees are 2 and 1 , so a polynomial basis matrix for $X_{R}$ is given by

$$
\left(\begin{array}{lll}
\lambda & 1 & 0 \\
0 & 0 & 1
\end{array}\right)
$$

We get $G=\left[\begin{array}{ll}I & 0\end{array}\right] \in \mathbb{R}^{3 \times 4}$. We now have to choose $\tilde{B}$ to complete $B(\infty)$ to an invertible matrix; we can take $\tilde{B}=\left[\begin{array}{lll}0 & 0 & 1\end{array}\right]$, which gives

$$
\hat{B}(\infty)=\left(\begin{array}{lll}
1 & 1 & 0 \\
0 & 1 & 0 \\
0 & 0 & 1
\end{array}\right)
$$

so that

$$
\hat{B}(\infty)^{-1}=\left(\begin{array}{rrr}
1 & -1 & 0 \\
0 & 1 & 0 \\
0 & 0 & 1
\end{array}\right)
$$

Therefore,

$$
H=\left(\begin{array}{rrr}
1 & -1 & 0 \\
0 & 1 & 0 \\
0 & 0 & 1
\end{array}\right)\left(\begin{array}{llll}
1 & 0 & 0 & 0 \\
0 & 0 & 1 & 0 \\
0 & 0 & 0 & 1
\end{array}\right)=\left(\begin{array}{rrrr}
1 & 0 & -1 & 0 \\
0 & 0 & 1 & 0 \\
0 & 0 & 0 & 1
\end{array}\right)
$$


Finally,

$$
\begin{aligned}
F(\lambda) & =\left(\begin{array}{rrrr}
\lambda^{2} & \lambda & 0 & 0 \\
0 & 0 & \lambda & 0
\end{array}\right)-\left(\begin{array}{rrr}
\lambda^{2} & \lambda^{2}+1 & 0 \\
1 & \lambda+2 & 3
\end{array}\right)\left(\begin{array}{rrrr}
1 & 0 & -1 & 0 \\
0 & 0 & 1 & 0 \\
0 & 0 & 0 & 1
\end{array}\right) \\
& =\left(\begin{array}{rrrr}
0 & \lambda & -1 & 0 \\
-1 & 0 & -1 & -3
\end{array}\right) .
\end{aligned}
$$

The matrix of $F$ is, therefore,

$$
F=\left(\begin{array}{rrrr}
0 & 1 & 0 & 0 \\
0 & 0 & -1 & 0 \\
-1 & 0 & -1 & -3
\end{array}\right)
$$

This concludes the example.

Now, suppose that a division of the external variables into inputs and outputs has been given, and that we want to obtain a representation in descriptor form. We start from the autoregressive equations, which appear in partitioned form:

$$
\left[\begin{array}{ll}
R_{1}(\sigma) & R_{2}(\sigma)
\end{array}\right]\left(\begin{array}{l}
y \\
u
\end{array}\right)=0
$$

Taking $R(s)$ to row proper form as before, we get a corresponding partitioning of the right bicausal matrix $B(s)$ :

$$
\left[R_{1}(s) \quad R_{2}(s)\right]=\Delta(s)\left[B_{1}(s) \quad B_{2}(s)\right] .
$$

By renumbering the inputs if necessary, we may assume that

$$
B_{2}(\infty)=\left[B_{2}^{1}(\infty) \quad B_{2}^{2}(\infty)\right]
$$

where $B_{2}^{1}(\infty)$ has full column rank, and the columns of $B_{2}^{2}(\infty)$ depend linearly on those of $\left[B_{1}(\infty) B_{2}^{1}(\infty)\right]$. Let $B_{2}^{2}(\infty)$ have $m_{2}$ columns; note that $m_{2} \leqq q-k$. It is easily verified that a matrix $\tilde{B}$ which completes $B(\infty)$ to an invertible matrix may be found whose last $m_{2}$ rows are in the form [0 $\left.I\right]$. By the construction, a basis matrix for $\operatorname{ker}\left[B_{1}(\infty) B_{2}^{1}(\infty)\right]$ must be of the form $\left[\begin{array}{ll}N & 0\end{array}\right]^{\top}$. Taking these facts together, we conclude that $\hat{B}(\infty)^{-1}$ is of the form

$$
\hat{B}(\infty)^{-1}=\left(\begin{array}{ccc}
* & * & * \\
* & 0 & * \\
0 & 0 & I
\end{array}\right)
$$

where the partitioning is $\left(p+m_{1}+m_{2}\right) \times\left(k+\left(q-k-m_{2}\right)+m_{2}\right)$ ( $p$ is the number of $y$-variables, $m_{1}$ is the number of columns of $\left.B_{2}^{1}(\infty)\right)$. We therefore obtain equations of the following form:

$$
\begin{gathered}
\sigma z_{0}=A_{0} z_{0}+B_{1} z_{1}+B_{2} z_{2} \\
y=H_{00} z_{0}+H_{01} z_{1}+H_{02} z_{2} \\
u_{1}=H_{10} z_{0}+H_{12} z_{2} \\
u_{2}=z_{2} .
\end{gathered}
$$

This can obviously be rewritten as

$$
\begin{gathered}
\sigma\left(\begin{array}{ll}
I & 0 \\
0 & 0
\end{array}\right)\left(\begin{array}{l}
z_{0} \\
z_{1}
\end{array}\right)=\left(\begin{array}{cc}
A_{0} & B_{1} \\
H_{10} & 0
\end{array}\right)\left(\begin{array}{l}
z_{0} \\
z_{1}
\end{array}\right)+\left(\begin{array}{cc}
0 & B_{2} \\
-I & H_{12}
\end{array}\right)\left(\begin{array}{l}
u_{1} \\
u_{2}
\end{array}\right) \\
y=\left[\begin{array}{ll}
H_{00} & H_{01}
\end{array}\right]\left(\begin{array}{l}
z_{0} \\
z_{1}
\end{array}\right)+\left[\begin{array}{ll}
0 & H_{02}
\end{array}\right]\left(\begin{array}{l}
u_{1} \\
u_{2}
\end{array}\right) .
\end{gathered}
$$


We now have a representation in descriptor form; as can be verified by checking the dimensions (using Remark 7.7), it is in fact a minimal representation. The fact that a zero block appears in the bottom right corner of the " $A$-matrix" means that the system "has no nondynamic variables" ([17]). It will be shown in [9] that the absence of nondynamic variables is a necessary condition for minimality of descriptor representations under external equivalence.

Example 8.2. Take

$$
R(s)=\left(\begin{array}{cccc}
s+1 & 0 & s^{2} & 2 \\
s+2 & 2 s & 1 & s-1
\end{array}\right)
$$

and let the first two external variables be outputs, and the other two inputs. The leading row coefficient matrix

$$
\left(\begin{array}{llll}
0 & 0 & 1 & 0 \\
1 & 2 & 0 & 1
\end{array}\right)
$$

has full row rank, so that the given matrix $R(s)$ is already row reduced; also, $m_{2}=1$ and the inputs need not be renumbered. We see that the sum of the row indices of $R(s)$ is 3 and that the row and the column defects of $B_{1}(\infty)$ (formed by the first two columns of the matrix above) are both equal to 1 ; so, a descriptor representation ( $E$, $A, B, C, D)$ will be minimal if and only if the matrix $E$ has size $4 \times 4$ and rank 3 .

We can take

$$
\tilde{B}=\left(\begin{array}{llll}
1 & 0 & 0 & 0 \\
0 & 0 & 0 & 1
\end{array}\right)
$$

which leads to

$$
\hat{B}(\infty)^{-1}=\left(\begin{array}{cccc}
0 & 0 & 1 & 0 \\
0 & \frac{1}{2} & -\frac{1}{2} & -\frac{1}{2} \\
1 & 0 & 0 & 0 \\
0 & 0 & 0 & 1
\end{array}\right)
$$

Consequently, we get

$$
H=\left(\begin{array}{ccccc}
0 & 0 & 0 & 1 & 0 \\
0 & 0 & \frac{1}{2} & -\frac{1}{2} & -\frac{1}{2} \\
1 & 0 & 0 & 0 & 0 \\
0 & 0 & 0 & 0 & 1
\end{array}\right) .
$$

The matrix of $F$ is computed from

$$
\begin{aligned}
F(\lambda)= & \left(\begin{array}{ccccc}
\lambda^{2} & \lambda & 0 & 0 & 0 \\
0 & 0 & \lambda & 0 & 0
\end{array}\right)-\left(\begin{array}{cccc}
\lambda+1 & 0 & \lambda^{2} & 2 \\
\lambda+2 & 2 \lambda & 1 & \lambda-1
\end{array}\right) \\
& \times\left(\begin{array}{rrrcc}
0 & 0 & 0 & 1 & 0 \\
0 & 0 & \frac{1}{2} & -\frac{1}{2} & -\frac{1}{2} \\
1 & 0 & 0 & 0 & 0 \\
0 & 0 & 0 & 0 & 1
\end{array}\right) \\
= & \left(\begin{array}{rrrrr}
0 & \lambda & 0 & -\lambda-1 & -2 \\
-1 & 0 & 0 & 2 & 1
\end{array}\right) .
\end{aligned}
$$


This gives

$$
F=\left(\begin{array}{rrrrr}
0 & 1 & 0 & -1 & 0 \\
0 & 0 & 0 & -1 & -2 \\
-1 & 0 & 0 & 2 & 1
\end{array}\right)
$$

Of course, $G=\left[\begin{array}{ll}I_{3} & 0\end{array}\right]$. Reorganizing the pencil equations as described above, we obtain

$$
\begin{gathered}
\sigma\left(\begin{array}{cc}
I_{3} & 0 \\
0 & 0
\end{array}\right)\left(\begin{array}{l}
z_{0} \\
z_{1}
\end{array}\right)=\left(\begin{array}{rrrr}
0 & 1 & 0 & -1 \\
0 & 0 & 0 & -1 \\
-1 & 0 & 0 & 2 \\
1 & 0 & 0 & 0
\end{array}\right)\left(\begin{array}{c}
z_{0} \\
z_{1}
\end{array}\right)+\left(\begin{array}{rr}
0 & 0 \\
0 & -2 \\
0 & 1 \\
-1 & 0
\end{array}\right)\left(\begin{array}{l}
u_{1} \\
u_{2}
\end{array}\right) \\
y=\left(\begin{array}{llll}
0 & 0 & 0 & 1 \\
0 & 0 & \frac{1}{2} & -\frac{1}{2}
\end{array}\right)\left(\begin{array}{c}
z_{0} \\
z_{1}
\end{array}\right)+\left(\begin{array}{cc}
0 & 0 \\
0 & -\frac{1}{2}
\end{array}\right)\left(\begin{array}{l}
u_{1} \\
u_{2}
\end{array}\right)
\end{gathered}
$$

9. Conclusions. In this paper, we have shown a procedure which leads from a representation in autoregressive form (and in particular, from a left polynomial factorization) to a minimal descriptor representation. This procedure does not require the separation of finite and infinite frequencies. In fact, the transfer matrix is never computed, and the heaviest computational load in the algorithm consists of the inversion of a single constant matrix. The basic tool that we used is the pencil representation, which appears as a natural form that can be derived from autoregressive equations by a very simple formula. This formula also provides the link between the realization theory of Willems and that of Fuhrmann. The direct connection between autoregressive representations and descriptor representations which has now been established enables us to study more closely the relations between the two representations.

\section{REFERENCES}

[1] J. D. Aplevich, Time-domain input-output representations of linear systems, Automatica, 17 (1981), pp. 509-522.

[2] - Minimal representations of implicit linear systems, Automatica, 21 (1985), pp. 259-269.

[3] H. Blomberg and R. Ylinen, Algebraic Theory for Multivariable Linear Systems, Academic Press, London, 1983.

[4] G. CONTE AND A. M. PERDON, Generalized state-space realizations for non-proper rational transfer functions, Systems Control Lett., 1 (1982), pp. 270-276.

[5] P. A. Fuhrmann, Algebraic system theory: an analyst's point of view, J. Franklin Inst., 301 (1976), pp. 521-540.

[6] - Linear Systems and Operators in Hilbert Space, McGraw-Hill, New York, 1981.

[7] J. Grimm, Application de la théorie des systèmes implicites a l'inversion des systèmes, in Analysis and Optimization of Systems, A. Bensoussan and J. L. Lions, eds., Proc. 6th Int. Conf., Nice, June 1984; part 2, Lecture Notes Control Information Sciences 63, Springer-Verlag, Berlin, New York, 1984, pp. $142-156$.

[8] T. Kailath, Linear Systems, Prentice-Hall, Englewood Cliffs, NJ, 1980.

[9] M. KUIJPER AND J. M. SCHUMACHER, Minimality of descriptor representations under external equivalence, Report BS-R9002, CWI, Amsterdam, 1990.

[10] D. G. Luenberger, Dynamic equations in descriptor form, IEEE Trans. Automat. Control, AC-22, (1977), pp. 312-321.

[11] D. G. Luenberger and A. Arbel, Singular dynamic Leontief models, Econometrica, 45 (1978), pp. $473-481$.

[12] H. H. Rosenbrock, Structural properties of linear dynamical systems, Internat. J. Control, 20 (1974), pp. 191-202.

[13] H. H. Rosenbrock, Non-minimal LCR multiports, Internat. J. Control, 20 (1974), pp. 1-16.

[14] D. SALAMON, Infinite dimensional systems with unbounded control and observation: a functional analytic approach, Trans. AMS, 300 (1987), pp. 383-431. 
[15] J. M. SCHUMACHeR, Transformations of linear systems under external equivalence, Linear Algebra Appl., 102 (1988), pp. 1-34.

[16] P. VAN DOOREN, The generalized eigenstructure problem in linear system theory, IEEE Trans. Automat. Control, AC-26 (1981), pp. 111-129.

[17] G. C. Verghese, B. LÉVy, AND T. KaILATH, A generalized state space for singular systems, IEEE Trans. Automat. Control, AC-26 (1981), pp. 811-831.

[18] J. C. WILlems, Input-output and state-space representations of finite-dimensional linear time-invariant systems, Linear Algebra Appl., 50 (1983), pp. 581-608.

[19] - From time series to linear system. Part I: Finite dimensional linear time invariant systems, Automatica, 22 (1986), pp. 561-580.

[20] — From time series to linear system. Part II: Exact modelling, Automatica, 22 (1986), pp. 675-694.

[21] — - From time series to linear system. Part III: Approximate modelling, Automatica, 23 (1987), pp. $87-115$.

[22] H. K. WIMMER, The structure of nonsingular polynomial matrices, Math. Systems Theory, 14 (1981), pp. 367-379. 